\title{
Matrix Models from Operators and Topological Strings, 2
}

\author{
Rinat Kashaev, Marcos Mariño and Szabolcs Zakany
}

\begin{abstract}
The quantization of mirror curves to toric Calabi-Yau threefolds leads to trace class operators, and it has been conjectured that the spectral properties of these operators provide a non-perturbative realization of topological string theory on these backgrounds. In this paper, we find an explicit form for the integral kernel of the trace class operator in the case of local $\mathbb{P}^{1} \times \mathbb{P}^{1}$, in terms of Faddeev's quantum dilogarithm. The matrix model associated to this integral kernel is an $O(2)$ model, which generalizes the $\mathrm{ABJ}(\mathrm{M})$ matrix model. We find its exact planar limit, and we provide detailed evidence that its $1 / N$ expansion captures the all genus topological string free energy on local $\mathbb{P}^{1} \times \mathbb{P}^{1}$.
\end{abstract}

\section{Introduction}

Topological strings on Calabi-Yau (CY) manifolds, just like other string theories, are only defined in perturbation theory, in terms of a genus expansion. In the closed string sector, the topological string free energies compute the Gromov-Witten invariants of the CY target, and for this reason topological string theory has played a prominent role in the interface of string theory, geometry, and mathematical physics.

Recently, it has been conjectured in [1] that topological strings on toric CY threefolds are captured, non-perturbatively, by the spectral theory of quantum-mechanical, trace class operators. These operators arise naturally in the quantization of their mirror curves. The conjecture of [1] builds upon previous ideas on quantization and mirror symmetry [2-5], but it also incorporates many conceptual aspects of large $N$ dualities. In fact, many of the crucial ingredients in the proposal of [1] were first unveiled in the study of the ABJM matrix model at large $N[6-11] .{ }^{1}$ As spelled out in detail in [13], one way of

\footnotetext{
1 The approach of [10] was first applied to topological string theory in [12], but it requires
} corrections which are incorporated in [1]. 
formulating the spectral theory/mirror symmetry correspondence of [1] is by considering the so-called fermionic traces $Z(N, \hbar)$ of the trace class operator (see Sect. 2.1 for a precise definition.) It turns out that, in the 't Hooft limit,

$$
N \rightarrow \infty, \quad \hbar \rightarrow \infty, \quad \frac{N}{\hbar}=\lambda \text { fixed },
$$

these traces have an asymptotic expansion of the form,

$$
\log Z(N, \hbar)=\sum_{g \geq 0} \mathcal{F}_{g}(\lambda) \hbar^{2-2 g} .
$$

According to the conjecture of [1], the functions $\mathcal{F}_{g}(\lambda)$ should be the genus $g$ free energies of the standard topological string, in the so-called conifold frame. The 't Hooft parameter $\lambda$ is a flat coordinate for the CY moduli space and is given by the vanishing period at the conifold point (we are assuming here that the mirror curve has genus one.) In this way, the weakly coupled topological string emerges in a limit in which the quantum-mechanical problem is strongly coupled (since $\hbar \rightarrow \infty$ ). On the other hand, the double-scaling limit

$$
N \rightarrow \infty, \quad \hbar \rightarrow 0, \quad N \hbar=\mu \text { fixed }
$$

corresponds to the WKB expansion in the quantum-mechanical problem, and it is captured by the Nekrasov-Shatashvili (NS) limit of the refined topological string, in agreement with the results of $[4,5]$. An obvious corollary of (1.2) is that the fermionic spectral traces $Z(N, \hbar)$ of the trace class operator provide a non-perturbative definition of the topological string partition function, in the spirit of large $N$ dualities. From a more physical point of view, one can regard $Z(N, \hbar)$ as the canonical partition function of a quantum ideal gas of $N$ fermions, where the operator plays the role of density matrix [6].

As explained in [13], if the kernel of the operator arising in the quantization of the mirror curve is known explicitly, then the fermionic spectral traces can be computed by a matrix model. Fortunately, it was shown in [14] that, for some simple mirror curves (leading to so-called three-term operators), one can compute the corresponding kernels in closed form, in terms of Faddeev's quantum dilogarithm. This made it possible to verify the trace class property conjectured in [1]. Armed with these kernels, one can compute the fermionic spectral traces $Z(N, \hbar)$, which are given by a generalized $O(2)$ matrix model of the type considered in [15]. In [13] this matrix model was studied in the $1 / N$ expansion, and it was checked in detail that, for local $\mathbb{P}^{2}$ and a certain limit of local $\mathbb{F}_{2},(1.2)$ gives indeed the topological string free energies.

This paper extends the results of $[13,14]$ to an important local CY, namely local $\mathbb{P}^{1} \times \mathbb{P}^{1}$. In this case, the mirror curve has genus one, therefore one modulus, but it also involves a mass parameter, since the geometry has two Kähler parameters. The quantization of this curve leads to a four-term operator. By the quantum pentagon identity for Faddeev's quantum dilogarithm, we find an explicit expression for the integral kernel of the corresponding trace class operator. The matrix model obtained from this kernel turns out to be an $O(2)$ matrix model. We compute some spectral traces at finite $N$, as a function of the mass parameter, which agree with the predictions of the conjecture in 
[1], as shown in [16]. We also study the matrix model in the large $N$ limit. This can be done by doing perturbation theory in the 't Hooft coupling, as in [13], but we can also use the general techniques of $[17,18]$, as developed in [19], to solve exactly for its planar limit. We compare the resulting $1 / N$ expansion with the topological string genus expansion, and we find a detailed agreement.

It is known, geometrically, that the topological string on $\mathbb{P}^{1} \times \mathbb{P}^{1}$ is equivalent to the topological string on local $\mathbb{F}_{2}$ by a simple change of parameters [16] (this leads to a relation between the Gromov-Witten invariants of the two geometries, as pointed out in $[16,20])$. We show that this equivalence holds as a unitary equivalence between the corresponding trace class operators. This allows us to extend all of our results to local $\mathbb{F}_{2}$ with arbitrary moduli, extending in this way the analysis presented in [13].

The trace class operator obtained by quantization of the mirror curve of local $\mathbb{P}^{1} \times \mathbb{P}^{1}$ can be regarded as a generalization of the density matrix appearing in the Fermi gas formulation of the ABJ(M) matrix model [6,2123], after an analytic continuation to complex mass parameters. Therefore, one can rederive from our results various aspects of the $\mathrm{ABJ}(\mathrm{M})$ matrix model. For example, we show that the exact planar solution of the $O(2)$ matrix model reproduces the planar free energy of the $\mathrm{ABJ}(\mathrm{M})$ matrix model obtained in $[24]$.

It is natural to ask how our matrix model for local $\mathbb{P}^{1} \times \mathbb{P}^{1}$ compares to a previous proposal in [25]. This proposal is based on a generalization of the Gopakumar-Vafa large $N$ duality [26], in which topological string theory on local $\mathbb{P}^{1} \times \mathbb{P}^{1}$ is described by large $N, U(N)$ Chern-Simons theory on the lens space $L(2,1)[25]$. When this is combined with the results of [27], one obtains a matrix model description of topological strings on local $\mathbb{P}^{1} \times \mathbb{P}^{1}$ which has been studied in some detail $[25,28,29]$. There are, however, many important differences between these matrix models. First of all, the matrix model of [25] is a two-cut matrix model, while our model is a one-cut matrix model. This leads to important differences at the non-perturbative level, since in the model of [25] the two Kähler parameters of local $\mathbb{P}^{1} \times \mathbb{P}^{1}$ are discretized (they correspond to the two filling fractions of the two-cut matrix model), while in the matrix model described here only the "diagonal" Kähler parameter is discretized. Another difference between these two matrix models is that the weak 't Hooft coupling expansion of the model in [25] corresponds to the so-called orbifold point in the moduli space of local $\mathbb{P}^{1} \times \mathbb{P}^{1}$, while in the model considered here it corresponds to the conifold point. Both points lead to logarithmic periods (which are in fact needed to match the Gaussian behavior of the matrix models), but they are different. It would be interesting to understand in more detail the relationship between the two matrix models, specially at the non-perturbative level, but we will not pursue this problem here. ${ }^{2}$ Note that, if the conjecture of [1] is true, the matrix model description in terms of kernels of trace class operators studied in this paper is likely to apply to all toric CY threefolds. In contrast,

\footnotetext{
${ }^{2}$ We would like to thank R. Schiappa for raising this issue.
} 
the large $N$ duality of [25] applies only to a special type of geometries, obtained as ADE quotients of the resolved conifold.

This paper is organized as follows. In Sect. 2 we elaborate on [14] and obtain an explicit representation for the integral kernel of the trace class operator associated to local $\mathbb{P}^{1} \times \mathbb{P}^{1}$. We also write down an $O(2)$ matrix model computing the fermionic spectral traces, and we study its $1 / N$ expansion. We obtain perturbative results as well as a closed form expression for the planar free energy, which can be expanded at both weak and strong coupling. In addition, we show how many known results for the $\mathrm{ABJ}(\mathrm{M})$ matrix model can be recovered from this solution. In Sect. 3 we compare successfully the $1 / N$ expansion of the matrix model with the topological string free energies of local $\mathbb{P}^{1} \times \mathbb{P}^{1}$, which we compute around a generic point in the conifold locus. We conclude in Sect. 4 and we list some open problems for the future. In the Appendix, we list some properties of the quantum dilogarithm which are used in Sect. 2.

\section{Operators, Kernels and Matrix Models}

\subsection{Integral Kernel and Matrix Model for Local $\mathbb{P}^{\mathbf{1}} \times \mathbb{P}^{\mathbf{1}}$}

As explained in $[1,14]$, given the mirror curve to a toric CY threefold, one can quantize it to obtain a trace class operator $\rho$. Although this procedure can be followed for any toric geometry, the simpler case is that of toric (almost) del Pezzo CY threefolds, defined as the total space of the canonical line bundle on a toric (almost) del Pezzo surface $S$,

$$
X=\mathcal{O}\left(K_{S}\right) \rightarrow S .
$$

In this case, the mirror curve has genus one. The complex moduli of the curve involve a "true" geometric modulus $\tilde{u}$ as well as a set of "mass" parameters $m_{i}, i=1, \ldots, r$, where $r$ depends on the geometry under consideration [30,31]. The mirror curves can be put in the "canonical" form

$$
W\left(\mathrm{e}^{x}, \mathrm{e}^{y}\right)=\mathcal{O}_{S}(x, y)+\tilde{u}=0,
$$

where $\mathcal{O}_{S}(x, y)$ is given by

$$
\mathcal{O}_{S}(x, y)=\sum_{i=1}^{k+2} \exp \left(\nu_{1}^{(i)} x+\nu_{2}^{(i)} y+f_{i}\left(m_{j}\right)\right),
$$

and $f_{i}\left(m_{j}\right)$ are suitable functions of the parameters $m_{j}$. The vectors $\nu_{1,2}^{(i)}$ can be obtained from the toric description of the CY threefold. The mirror curve (2.2) is quantized by standard Weyl quantization. In particular, $x, y$ are promoted to self-adjoint Heisenberg operators $\mathrm{x}, \mathrm{y}$, satisfying the commutation relation

$$
[\mathrm{x}, \mathrm{y}]=\mathrm{i} \hbar \text {, }
$$

and ordering ambiguities are resolved by Weyl's prescription. In this way, $\mathcal{O}_{S}(x, y)$ becomes an operator, which will be denoted by $\mathrm{O}_{S}$. As conjectured in [1] and proved in [14] in many cases, the inverse operator 


$$
\rho_{S}=\mathrm{O}_{S}^{-1}
$$

is of trace class.

In this paper we will focus on the important local del Pezzo CY threefold in which $S=\mathbb{P}^{1} \times \mathbb{P}^{1}=\mathbb{F}_{0}$, and usually called local $\mathbb{P}^{1} \times \mathbb{P}^{1}$ or local $\mathbb{F}_{0}$. Topological string theory on this background is known to have various applications: it engineers geometrically $S U(2)$ Seiberg-Witten theory [32], it is dual to Chern-Simons theory on the lens space $L(2,1)[25]$, and it is closely related to the partition function of $\mathrm{ABJ}(\mathrm{M})$ theory on the three-sphere $[6,24]$. In this case, the function $\mathcal{O}_{S}(x, y)$ is given by,

$$
\mathcal{O}_{\mathbb{F}_{0}}(x, y)=\mathrm{e}^{x}+m_{\mathbb{F}_{0}} \mathrm{e}^{-x}+\mathrm{e}^{y}+\mathrm{e}^{-y},
$$

and depends on a mass parameter that we denote by $m_{\mathbb{F}_{0}}$. In principle, we will take $m_{\mathbb{F}_{0}}$ to be real and positive, but as we will see it is possible to extend some of the results to complex values of $m_{\mathbb{F}_{0}}$.

We would like to find an explicit expression for the kernel of the operator $\rho_{\mathbb{F}_{0}}$. As for the three-term operators analyzed in [14], this kernel will involve in a crucial way Faddeev's quantum dilogarithm $\Phi_{\mathrm{b}}(x)$ [33-35], see the Appendix for its definition and some of its basic properties. In addition, the function $\Phi_{\mathrm{b}}(x)$ has the following features. If $\mathrm{p}$ and $\mathrm{q}$ are self-adjoint Heisenberg operators satisfying,

$$
[\mathrm{p}, \mathrm{q}]=(2 \pi \mathrm{i})^{-1},
$$

the quantum dilogarithm satisfies [14]

$$
\begin{aligned}
\Phi_{b}(p) e^{2 \pi b q} \Phi_{b}^{*}(p) & =e^{2 \pi b q}+e^{2 \pi b(p+q)}, \\
\Phi_{b}^{*}(q) \Phi_{b}(p) e^{2 \pi b q} \Phi_{b}^{*}(p) \Phi_{b}(q) & =e^{2 \pi b q}+e^{2 \pi b(p+q)}+e^{2 \pi b(p+2 q)} .
\end{aligned}
$$

One also has the important quantum pentagon identity [36],

$$
\Phi_{b}(p) \Phi_{b}(q)=\Phi_{b}(q) \Phi_{b}(p+q) \Phi_{b}(p) .
$$

The quantization of (2.6) leads to the operator,

$$
\mathrm{O}_{\mathbb{F}_{0}}=\mathrm{e}^{\mathrm{x}}+m_{\mathbb{F}_{0}} \mathrm{e}^{-\mathrm{x}}+\mathrm{e}^{\mathrm{y}}+\mathrm{e}^{-\mathrm{y}} .
$$

Let us set

$$
\hbar=\pi \mathrm{b}^{2}
$$

and

$$
\mathrm{x}=\pi \mathrm{b}(\mathrm{p}+2 \mathrm{q}), \quad \mathrm{y}=\pi \mathrm{bp}
$$

Using (2.8), we find

$$
\begin{aligned}
\mathrm{e}^{\mathrm{x} / 2} \mathrm{O}_{\mathbb{F}_{0}} \mathrm{e}^{\mathrm{x} / 2}-m_{\mathbb{F}_{0}} & =\mathrm{e}^{2 \mathrm{x}}+\mathrm{e}^{\mathrm{x}+\mathrm{y}}+\mathrm{e}^{\mathrm{x}-\mathrm{y}}=\mathrm{e}^{2 \pi \mathrm{b}(\mathrm{p}+2 \mathrm{q})}+\mathrm{e}^{2 \pi \mathrm{b}(\mathrm{p}+\mathrm{q})}+\mathrm{e}^{2 \pi \mathrm{bq}} \\
& =\Phi_{\mathrm{b}}^{*}(q) \Phi_{\mathrm{b}}(\mathrm{p}) \mathrm{e}^{2 \pi \mathrm{bq}} \Phi_{\mathrm{b}}^{*}(\mathrm{p}) \Phi_{\mathrm{b}}(\mathrm{q})
\end{aligned}
$$


Therefore,

$$
\begin{aligned}
\Phi_{\mathrm{b}}^{*}(\mathrm{p}) \Phi_{\mathrm{b}}(\mathrm{q}) \mathrm{e}^{\mathrm{x} / 2} \mathrm{O}_{\mathbb{F}_{0}} \mathrm{e}^{\mathrm{x} / 2} \Phi_{\mathrm{b}}^{*}(\mathrm{q}) \Phi_{\mathrm{b}}(\mathrm{p}) & =m_{\mathbb{F}_{0}}+\mathrm{e}^{2 \pi \mathrm{bq}} \\
& =m_{\mathbb{F}_{0}}\left(1+\mathrm{e}^{2 \pi \mathrm{b}(\mathrm{q}-\mathrm{b} \xi / \pi)}\right) \\
& =m_{\mathbb{F}_{0}} \frac{\Phi_{\mathrm{b}}(\mathrm{q}-\mathrm{b} \xi / \pi-\mathrm{ib} / 2)}{\Phi_{\mathrm{b}}(\mathrm{q}-\mathrm{b} \xi / \pi+\mathrm{ib} / 2)},
\end{aligned}
$$

where the parameter $\xi$ is related to $m_{\mathbb{F}_{0}}$ through the equation

$$
m_{\mathbb{F}_{0}}=\mathrm{e}^{2 \mathrm{~b}^{2} \xi} \text {. }
$$

Let us now define the operator

$$
\mathrm{B} \equiv \Phi_{\mathrm{b}}^{*}(\mathrm{q}-\mathrm{b} \xi / \pi-\mathrm{ib} / 2) \Phi_{\mathrm{b}}^{*}(\mathrm{p}) \Phi_{\mathrm{b}}(\mathrm{q}) \mathrm{e}^{\pi \mathrm{bp} / 2} \mathrm{e}^{\pi \mathrm{bq}} .
$$

We obtain the following formula [14]

$$
\mathrm{O}_{\mathbb{F}_{0}}^{-1}=m_{\mathbb{F}_{0}}^{-1} \mathrm{~B}^{*} \mathrm{~B} .
$$

On the other hand, we can use the quantum pentagon relation (2.9) to write the operator B as

$$
\begin{aligned}
& \mathrm{e}^{-(\pi \mathrm{b} / 2)^{2} /(4 \pi \mathrm{i})} \Phi_{\mathrm{b}}(\mathrm{p}) \mathrm{B} \\
& =\Phi_{\mathrm{b}}^{*}(\mathrm{p}+\mathrm{q}-\mathrm{b} \xi / \pi-\mathrm{ib} / 2) \Phi_{\mathrm{b}}^{*}(\mathrm{q}-\mathrm{b} \xi / \pi-\mathrm{ib} / 2) \Phi_{\mathrm{b}}(\mathrm{q}) \mathrm{e}^{\pi \mathrm{b}(\mathrm{p}+\mathrm{q}) / 2} \mathrm{e}^{\pi \mathrm{bq} / 2} \\
& =\Phi_{\mathrm{b}}^{*}(\mathrm{p}+\mathrm{q}-\mathrm{b} \xi / \pi-\mathrm{ib} / 2) \mathrm{e}^{\pi \mathrm{b}(\mathrm{p}+\mathrm{q}) / 2} \Phi_{\mathrm{b}}^{*}(\mathrm{q}-\mathrm{b} \xi / \pi-i \mathrm{~b} / 4) \Phi_{\mathrm{b}}(\mathrm{q}+\mathrm{ib} / 4) \mathrm{e}^{\pi \mathrm{bq} / 2} .
\end{aligned}
$$

If we introduce new momentum and position operators by

$$
\mathrm{p}^{\prime} \equiv \mathrm{p}+\mathrm{q}-\mathrm{b} \xi / \pi, \quad \mathrm{q}^{\prime} \equiv \mathrm{q}-\mathrm{b} \xi / 2 \pi
$$

we find

$$
\rho_{\mathbb{F}_{0}}=\mathrm{e}^{-\mathrm{b}^{2} \xi / 2} f\left(\mathrm{q}^{\prime}\right) \frac{1}{2 \cosh \left(\pi \mathrm{bp}^{\prime}\right)} f^{*}\left(\mathrm{q}^{\prime}\right)
$$

where

$$
f(q)=\mathrm{e}^{\pi \mathrm{bq} / 2} \frac{\Phi_{\mathrm{b}}(\mathrm{q}-\mathrm{b} \xi / 2 \pi+\mathrm{ib} / 4)}{\Phi_{\mathrm{b}}(\mathrm{q}+\mathrm{b} \xi / 2 \pi-\mathrm{ib} / 4)} .
$$

In the position representation for the operators $\mathrm{p}^{\prime}$ and $\mathrm{q}^{\prime}$, we obtain the integral kernel,

$$
\rho_{\mathbb{F}_{0}}\left(x_{1}, x_{2}\right)=\left\langle x_{1}\left|\mathrm{O}_{\mathbb{F}_{0}}^{-1}\right| x_{2}\right\rangle=\mathrm{e}^{-\mathrm{b}^{2} \xi / 2} \frac{f\left(x_{1}\right) f^{*}\left(x_{2}\right)}{2 \mathrm{~b} \cosh \left(\pi \frac{x_{1}-x_{2}}{\mathrm{~b}}\right)} .
$$

As shown already in [14], this is a positive-definite, trace class operator on $L^{2}(\mathbb{R})$. Note that $\rho_{\mathbb{F}_{0}}\left(x_{1}, x_{2}\right)$ is related by a unitary transformation to the symmetric, real kernel

$$
\mathrm{e}^{-\mathrm{b}^{2} \xi / 2} \frac{\left|f\left(x_{1}\right)\right|\left|f\left(x_{2}\right)\right|}{2 \mathrm{~b} \cosh \left(\pi \frac{x_{1}-x_{2}}{\mathrm{~b}}\right)},
$$

which is of the type considered in $[37,38]$. In particular, as shown in these references, its diagonal resolvent can be obtained from a TBA-like system of non-linear integral equations. 
The spectral information of a trace class operator $\rho$ depending on a parameter $\hbar$ and acting on a Hilbert space $\mathcal{H}$ can be encoded in different ways. The spectral traces of $\rho$ are defined by

$$
Z_{\ell}=\operatorname{Tr}_{\mathcal{H}} \rho^{\ell}, \quad \ell=1,2, \ldots
$$

The fermionic spectral traces are given by

$$
Z(N, \hbar)=\operatorname{Tr}_{\Lambda^{N}(\mathcal{H})}\left(\Lambda^{N}(\rho)\right), \quad N=1,2, \ldots
$$

where the operator $\Lambda^{N}(\rho)$ is defined by $\rho^{\otimes N}$ acting on $\Lambda^{N}(\mathcal{H})$. The generating function of the fermionic spectral traces is the Fredholm or spectral determinant of $\rho$ :

$$
\Xi(\kappa, \hbar)=\operatorname{det}(1+\kappa \rho)=1+\sum_{N=1}^{\infty} Z(N, \hbar) \kappa^{N},
$$

and it is an entire function of $\kappa$ due to the trace class property of $\rho$ [39].

A well-known theorem of Fredholm (see chapter 3 of [39] for a proof) states that $Z(N, \hbar)$ has the matrix model-like representation

$$
Z(N, \hbar)=\frac{1}{N !} \int \mathrm{d}^{N} x \operatorname{det}\left(\rho\left(x_{i}, x_{j}\right)\right) .
$$

In this equation, $\rho\left(x_{1}, x_{2}\right)$ is the integral kernel of the operator $\rho$,

$$
\rho\left(x_{1}, x_{2}\right)=\left\langle x_{1}|\rho| x_{2}\right\rangle .
$$

The spectral traces (2.24) and the fermionic spectral traces are closely related, since one has that

$$
\mathcal{J}(\kappa)=\log \Xi(\kappa, \hbar)=-\sum_{\ell=1}^{\infty} \frac{Z_{\ell}}{\ell}(-\kappa)^{\ell} .
$$

The above quantities can be interpreted, more physically, in terms of an ideal Fermi gas of $N$ particles, as in [6]. In this setting, $\rho$ is the canonical density matrix, $Z(N, \hbar)$ is the canonical partition function of the gas, $\Xi(\kappa, \hbar)$ is the grand canonical partition function, and $\mathcal{J}(\kappa)$ is the grand potential.

Since we have an explicit formula for the integral kernel of $\rho_{\mathbb{F}_{0}}$, we can write down an explicit expression for the integral (2.27). By Cauchy's identity, as in $[6,13,40]$,

$$
\begin{aligned}
\frac{\prod_{i<j}\left[2 \sinh \left(\frac{\mu_{i}-\mu_{j}}{2}\right)\right]\left[2 \sinh \left(\frac{\nu_{i}-\nu_{j}}{2}\right)\right]}{\prod_{i, j} 2 \cosh \left(\frac{\mu_{i}-\nu_{j}}{2}\right)} & =\operatorname{det}_{i j} \frac{1}{2 \cosh \left(\frac{\mu_{i}-\nu_{j}}{2}\right)} \\
& =\sum_{\sigma \in S_{N}}(-1)^{\epsilon(\sigma)} \prod_{i} \frac{1}{2 \cosh \left(\frac{\mu_{i}-\nu_{\sigma(i)}}{2}\right)}
\end{aligned}
$$


we obtain the following matrix model representation for the fermionic traces of $\rho_{\mathbb{F}_{0}}$,

$$
Z_{\mathbb{F}_{0}}(N, \hbar)=\frac{\mathrm{e}^{-\mathrm{b}^{2} \xi N / 2}}{N !} \int \frac{\mathrm{d}^{N} u}{(2 \pi)^{N}} \prod_{i=1}^{N}\left|f\left(\frac{\mathrm{b} u_{i}}{2 \pi}\right)\right|^{2} \frac{\prod_{i<j} 4 \sinh ^{2}\left(\frac{u_{i}-u_{j}}{2}\right)}{\prod_{i, j} 2 \cosh \left(\frac{u_{i}-u_{j}}{2}\right)},
$$

where the variables $u_{i}$ are related to the original variables $x_{i}$ by

$$
u_{i}=\frac{2 \pi}{\mathrm{b}} x_{i}
$$

\subsection{Relation to Local $\mathbb{F}_{2}$ and Spectral Traces}

It is known that topological string theory on the local $\mathbb{F}_{2}$ geometry is closely related to topological string theory on local $\mathbb{F}_{0}[16]$. It turns out that this equivalence also holds at the level of the corresponding quantum operators. To see this, let us first redefine the operators appearing in (2.10) as,

$$
\hbar=2 \pi \mathrm{b}^{2}, \quad \mathrm{x}=2 \pi \mathrm{bq}, \quad \mathrm{y}=2 \pi \mathrm{bp} .
$$

We then have,

$$
\begin{aligned}
\mathrm{e}^{\mathrm{x}}+\mathrm{e}^{\mathrm{y}}=\mathrm{e}^{\mathrm{x} / 2}\left(1+\mathrm{e}^{\mathrm{y}-\mathrm{x}}\right) \mathrm{e}^{\mathrm{x} / 2} & =\mathrm{e}^{\pi \mathrm{bq}} \frac{\Phi_{\mathrm{b}}(\mathrm{p}-\mathrm{q}-\mathrm{ib} / 2)}{\Phi_{\mathrm{b}}(\mathrm{p}-\mathrm{q}+\mathrm{ib} / 2)} \mathrm{e}^{\pi \mathrm{bq}} \\
& =\Phi_{\mathrm{b}}(\mathrm{p}-\mathrm{q}) \mathrm{e}^{2 \pi \mathrm{bq}} \Phi_{\mathrm{b}}(\mathrm{p}-\mathrm{q})^{-1}
\end{aligned}
$$

Therefore,

$$
\begin{aligned}
& \frac{1}{\Phi_{\mathrm{b}}(\mathrm{p}-\mathrm{q})} \mathrm{O}_{\mathbb{F}_{0}} \Phi_{\mathrm{b}}(\mathrm{p}-\mathrm{q})-\mathrm{e}^{2 \pi \mathrm{bq}} \\
& =\frac{1}{\Phi_{\mathrm{b}}(\mathrm{p}-\mathrm{q})}\left(m_{\mathbb{F}_{0}} \mathrm{e}^{-2 \pi \mathrm{bq}}+\mathrm{e}^{-2 \pi \mathrm{bp}}\right) \Phi_{\mathrm{b}}(\mathrm{p}-\mathrm{q}) \\
& \quad=m_{\mathbb{F}_{0}} \mathrm{e}^{-\pi \mathrm{bq}} \frac{\Phi_{\mathrm{b}}(\mathrm{p}-\mathrm{q}-\mathrm{ib} / 2)}{\Phi_{\mathrm{b}}(\mathrm{p}-\mathrm{q}+\mathrm{ib} / 2)} \mathrm{e}^{-\pi \mathrm{bq}}+\mathrm{e}^{-\pi \mathrm{bp}} \frac{\Phi_{\mathrm{b}}(\mathrm{p}-\mathrm{q}-\mathrm{ib} / 2)}{\Phi_{\mathrm{b}}(\mathrm{p}-\mathrm{q}+\mathrm{ib} / 2)} \mathrm{e}^{-\pi \mathrm{bp}} \\
& =m_{\mathbb{F}_{0}}\left(\mathrm{e}^{-2 \pi \mathrm{bq}}+\mathrm{e}^{2 \pi \mathrm{b}(\mathrm{p}-2 \mathrm{q})}\right)+\mathrm{e}^{-2 \pi \mathrm{bp}}+\mathrm{e}^{-2 \pi \mathrm{bq}} \\
& =\left(1+m_{\mathbb{F}_{0}}\right) \mathrm{e}^{-2 \pi \mathrm{bq}}+m_{\mathbb{F}_{0}} \mathrm{e}^{2 \pi \mathrm{b}(\mathrm{p}-2 \mathrm{q})}+\mathrm{e}^{-2 \pi \mathrm{bp}},
\end{aligned}
$$

or in terms of original variables

$$
\frac{1}{\Phi_{\mathrm{b}}(\mathrm{p}-\mathrm{q})} \mathrm{O}_{\mathbb{F}_{0}} \Phi_{\mathrm{b}}(\mathrm{p}-\mathrm{q})=\mathrm{e}^{\mathrm{x}}+\left(1+m_{\mathbb{F}_{0}}\right) \mathrm{e}^{-\mathrm{x}}+m_{\mathbb{F}_{0}} \mathrm{e}^{\mathrm{y}-2 \mathrm{x}}+\mathrm{e}^{-\mathrm{y}} .
$$

By defining new variables

$$
\mathrm{x}^{\prime}=\mathrm{x}+\nu, \quad \mathrm{y}^{\prime}=\mathrm{y}-2 \mathrm{x}-3 \nu, \quad \nu=-\frac{1}{4} \log \left(m_{\mathbb{F}_{0}}\right),
$$

we rewrite (2.36) as follows

$$
\frac{1}{\Phi_{\mathrm{b}}(\mathrm{p}-\mathrm{q})} m_{\mathbb{F}_{0}}^{-1 / 4} \mathrm{O}_{\mathbb{F}_{0}} \Phi_{\mathrm{b}}(\mathrm{p}-\mathrm{q})=\mathrm{e}^{\mathrm{x}^{\prime}}+\left(m_{\mathbb{F}_{0}}^{1 / 2}+m_{\mathbb{F}_{0}}^{-1 / 2}\right) \mathrm{e}^{-\mathrm{x}^{\prime}}+\mathrm{e}^{\mathrm{y}^{\prime}}+\mathrm{e}^{-2 \mathrm{x}^{\prime}-\mathrm{y}^{\prime}} .
$$


We conclude that the operator $m_{\mathbb{F}_{0}}^{-1 / 4} \mathrm{O}_{\mathbb{F}_{0}}$ is unitarily equivalent to the operator

$$
\mathrm{O}_{\mathbb{F}_{2}}=\mathrm{e}^{\mathrm{x}}+m_{\mathbb{F}_{2}} \mathrm{e}^{-\mathrm{x}}+\mathrm{e}^{\mathrm{y}}+\mathrm{e}^{-2 \mathrm{x}-\mathrm{y}},
$$

corresponding to the local $\mathbb{F}_{2}$ geometry $[1,14]$, after the substitution

$$
m_{\mathbb{F}_{2}}=m_{\mathbb{F}_{0}}^{1 / 2}+m_{\mathbb{F}_{0}}^{-1 / 2} \text {. }
$$

In the CY geometries, the rescaling by $m_{\mathbb{F}_{0}}^{-1 / 4}$ leads, in view of $(2.2)$, to the following relation between the moduli,

$$
\tilde{u}_{\mathbb{F}_{2}}=m_{\mathbb{F}_{0}}^{-1 / 4} \tilde{u}_{\mathbb{F}_{0}}
$$

The relationships (2.40), (2.41) agree precisely with those found by a direct analysis of the topological string in these geometries [16]. This means in particular that any test of the conjecture of [1] for local $\mathbb{F}_{0}$ leads automatically to a corresponding test for local $\mathbb{F}_{2}$. The unitary equivalence of the two operators also leads to the following equality of spectral traces,

$$
\operatorname{Tr} \rho_{\mathbb{F}_{2}}^{\ell}\left(m_{\mathbb{F}_{2}}\right)=m_{\mathbb{F}_{0}}^{\ell / 4} \operatorname{Tr} \rho_{\mathbb{F}_{0}}^{\ell}\left(m_{\mathbb{F}_{0}}\right),
$$

after the substitution (2.40).

Using the expression for the integral kernel in (2.22), as well as (2.42), we can in principle compute explicitly the first spectral traces. According to [1], we should expect simplifications in the so-called maximally supersymmetric case $\hbar=2 \pi$, which corresponds to

$$
\mathrm{b}=\sqrt{2}
$$

in (2.11). For this value of $b$, we can use the functional equation (A.9b) satisfied by the quantum dilogarithm to obtain the following expression in terms of elementary functions,

$$
|f(x)|^{2}=\frac{1}{4 \cosh \left(\frac{\pi \sqrt{2}(x-\mathrm{b} \xi / 2 \pi)}{2}\right) \cosh \left(\frac{\pi \sqrt{2}(x+\mathrm{b} \xi / 2 \pi)}{2}\right)} .
$$

After an appropriate change of variables, we obtain,

$$
\operatorname{Tr} \rho_{\mathbb{F}_{0}}=\frac{1}{8 \pi} m_{\mathbb{F}_{0}}^{-1 / 4} \int_{-\infty}^{\infty} \frac{\mathrm{d} u}{\cosh (u) \cosh (u-\sqrt{2} \mathrm{~b} \xi / 2)}=\frac{1}{8 \pi} \frac{\log \left(m_{\mathbb{F}_{0}}\right)}{m_{\mathbb{F}_{0}}^{1 / 2}-1} .
$$

The second trace is a little bit more complicated. We find

$$
\begin{aligned}
& \operatorname{Tr} \rho_{\mathbb{F}_{0}}^{2}=\frac{1}{64 \pi^{2}} m_{\mathbb{F}_{0}}^{-1 / 2} \\
& \int_{-\infty}^{\infty} \int_{-\infty}^{\infty} \frac{\mathrm{d} u \mathrm{~d} v}{\cosh (u) \cosh (u-\sqrt{2} \mathrm{~b} \xi / 2 \pi) \cosh (v) \cosh (v-\sqrt{2} \mathrm{~b} \xi / 2 \pi) \cosh (u-v)^{2}} \\
& =\frac{m_{\mathbb{F}_{0}}^{-1 / 2}}{16 \pi^{2}}\left[\left(\frac{\log \left(m_{\mathbb{F}_{0}}\right)}{m_{\mathbb{F}_{0}}^{1 / 2}-m_{\mathbb{F}_{0}}^{-1 / 2}}+1\right)^{2}-1-\frac{\pi^{2}}{\left(m_{\mathbb{F}_{0}}^{1 / 4}+m_{\mathbb{F}_{0}}^{-1 / 4}\right)^{2}}\right]
\end{aligned}
$$

When $m_{\mathbb{F}_{0}}=1$, these expressions give,

$$
\operatorname{Tr} \rho_{\mathbb{F}_{0}}\left(m_{\mathbb{F}_{0}}=1\right)=\frac{1}{4 \pi}, \quad \operatorname{Tr} \rho_{\mathbb{F}_{0}}^{2}\left(m_{\mathbb{F}_{0}}=1\right)=\frac{12-\pi^{2}}{64 \pi^{2}} .
$$


in accord with the values predicted in [1] from the spectral theory/mirror symmetry correspondence.

We can now use (2.42) to obtain the values of the same traces for local $\mathbb{F}_{2}$. We find,

$$
\begin{aligned}
\operatorname{Tr} \rho_{\mathbb{F}_{2}} & =\frac{1}{4 \pi} \frac{\cosh ^{-1}\left(m_{\mathbb{F}_{2}} / 2\right)}{\sqrt{m_{\mathbb{F}_{2}}-2}}, \\
\operatorname{Tr} \rho_{\mathbb{F}_{2}}^{2} & =\frac{1}{16 \pi^{2}}\left[\left(2 \frac{\cosh ^{-1}\left(m_{\mathbb{F}_{2}} / 2\right)}{\sqrt{m_{\mathbb{F}_{2}}^{2}-4}+1}\right)^{2}-1-\frac{\pi^{2}}{m_{\mathbb{F}_{2}}+2}\right] .
\end{aligned}
$$

We obtain, in particular

$$
\begin{aligned}
& \operatorname{Tr} \rho_{\mathbb{F}_{2}}\left(m_{\mathbb{F}_{2}}=0\right)=\frac{1}{8 \sqrt{2}}, \\
& \operatorname{Tr} \rho_{\mathbb{F}_{2}}^{2}\left(m_{\mathbb{F}_{2}}=0\right)=\frac{1}{64}\left(\frac{4}{\pi}-1\right),
\end{aligned}
$$

which were already obtained in [14], and

$$
\begin{aligned}
& \operatorname{Tr} \rho_{\mathbb{F}_{2}}\left(m_{\mathbb{F}_{2}}=1\right)=\frac{1}{12}, \\
& \operatorname{Tr} \rho_{\mathbb{F}_{2}}^{2}\left(m_{\mathbb{F}_{2}}=1\right)=\frac{1}{432}\left(\frac{12 \sqrt{3}}{\pi}-5\right) .
\end{aligned}
$$

It can be verified [16] that these values agree with the predictions of the conjecture in [1].

\subsection{Perturbative Expansion}

We are now interested in studying the matrix integral (2.31) in the 't Hooft limit (1.1). As in [13], we should first analyze the integrand of (2.31) when $\hbar$ (or equivalently b) is large. At the same time, we have to decide what is the appropriate scaling of the parameter $m_{\mathbb{F}_{0}}$ appearing in the operator, as $\hbar$ becomes large. As it was explained in [13], to recover the topological string for arbitrary mass parameter, we have to scale

$$
\log m_{\mathbb{F}_{0}} \sim \hbar, \quad \hbar \rightarrow \infty .
$$

We recall the variable $\xi$ is defined as

$$
\xi=\frac{\pi}{2 \hbar} \log m_{\mathbb{F}_{0}} .
$$

This is the mass variable that will be kept fixed in the 't Hooft limit. If we introduce the parameter

$$
\mathrm{g}=\frac{1}{\hbar}
$$


we can write the matrix integral (2.31) in the form

$$
Z(N, \hbar)=\frac{\mathrm{e}^{-\xi \lambda /\left(2 \pi \mathrm{g}^{2}\right)}}{N !} \int_{\mathbb{R}^{N}} \frac{\mathrm{d}^{N} u}{(2 \pi)^{N}} \prod_{i=1}^{N} \mathrm{e}^{-\frac{1}{\mathrm{~g}} V\left(u_{i}, \mathrm{~g}\right)} \frac{\prod_{i<j} 4 \sinh \left(\frac{u_{i}-u_{j}}{2}\right)^{2}}{\prod_{i, j} 2 \cosh \left(\frac{u_{i}-u_{j}}{2}\right)},
$$

where

$$
V(u, \mathrm{~g})=-\mathrm{g} \log \left|f\left(\frac{\mathrm{b} u}{2 \pi}\right)\right|^{2} .
$$

As in [13], we can now use the self-duality of Faddeev's quantum dilogarithm,

$$
\Phi_{\mathrm{b}}(x)=\Phi_{1 / \mathrm{b}}(x),
$$

as well as (A.10), to obtain the following asymptotic expansion for small $\mathrm{g}$,

$$
\begin{aligned}
V(u, \mathrm{~g}) \sim & -\frac{u}{2 \pi}-\frac{1}{\pi^{2}} \sum_{k \geq 0}\left(-4 \pi^{4} \mathrm{~g}^{2}\right)^{k} \frac{B_{2 k}(1 / 2)}{(2 k) !} \\
& \times \operatorname{Im}\left[\operatorname{Li}_{2-2 k}\left(-\mathrm{ie}^{u+\xi}\right)+\operatorname{Li}_{2-2 k}\left(-\mathrm{ie}^{u-\xi}\right)\right] .
\end{aligned}
$$

If we write this expansion as

$$
V(u, \mathrm{~g})=\sum_{\ell \geq 0} \mathrm{~g}^{2 \ell} V^{(\ell)}(u),
$$

we find that the leading contribution as $\mathrm{g} \rightarrow 0$ is given by the "classical" potential,

$$
V^{(0)}(u)=-\frac{u}{2 \pi}-\frac{1}{\pi^{2}}\left(\operatorname{Im} \operatorname{Li}_{2}\left(-\mathrm{i} \mathrm{e}^{u+\xi}\right)+\operatorname{Im}_{\operatorname{Li}}\left(-\mathrm{i} \mathrm{e}^{u-\xi}\right)\right) .
$$

The matrix integral (2.54) is an $O(2)$ matrix model [41], in which the inverse Planck constant g plays the role of the string coupling constant, and the potential itself depends on $g$. To obtain the 't Hooft expansion of the free energy, we can use the asymptotic expansion of the potential (2.58). In particular, since this expansion only involves even powers of $\hbar$, we conclude that the matrix integral (2.54) admits a standard 't Hooft expansion, of the form

$$
F(N, \hbar)=\log Z(N, \hbar)=\sum_{g \geq 0} \hbar^{2-2 g} \mathcal{F}_{g}(\lambda, \xi),
$$

where $\lambda$ is the " $t$ Hooft parameter introduced in (1.1), and $\xi$ was introduced in (2.52). Note that, in the planar limit, only the classical part of the potential (2.59) contributes. By the asymptotics of the dilogarithm, one finds that the classical potential behaves as

$$
V^{(0)}(u) \approx \frac{|u|}{2 \pi}, \quad|u| \rightarrow \infty
$$

i.e., it is a linearly confining potential at infinity, similar to the potentials appearing in matrix models for Chern-Simons-matter theories $[6,19]$ and in other matrix integrals associated to quantized mirror curves [13]. The potential (2.59), for two values of $\xi$, as well as its asymptotic form (2.61), is shown in Fig. 1. 


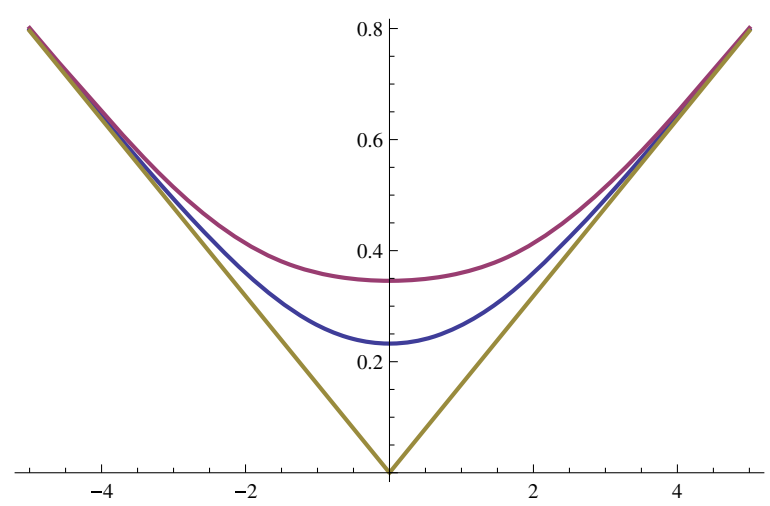

Figure 1. The classical potential (2.59) as a function of $u$, for $\xi=1$ (lower line) and $\xi=2$ (upper line), together with their asymptotic form (2.61) when $u$ is large

We would like to compute the genus $g$ free energies $\mathcal{F}_{g}(\lambda, \xi)$ appearing in the expansion (2.60). We will first obtain approximate expressions for the very first free energies, as expansions around $\lambda=0$, by doing perturbation theory in g, as in [13]. To do this, we regard (2.54) as a Gaussian Hermitian matrix model, perturbed by single and double trace operators. The computation is straightforward (see for example [25] for a similar example). For the genus $g$ free energies we find the following structure,

$$
\begin{aligned}
& \mathcal{F}_{0}(\lambda, \xi)=\frac{\lambda^{2}}{2}\left(\log \left(\frac{\pi^{2} \lambda \cosh \xi}{4}\right)-\frac{3}{2}\right)-\frac{2}{\pi^{2}} \operatorname{Im}\left(\operatorname{Li}_{2}\left(\mathrm{i} \mathrm{e}^{\xi}\right)\right) \lambda+\sum_{k \geq 3} f_{0, k} \lambda^{k} \\
& \mathcal{F}_{1}(\lambda, \xi)=-\frac{1}{12} \log \hbar-\frac{1}{12} \log \lambda+\zeta^{\prime}(-1)+\sum_{k \geq 1} f_{1, k} \lambda^{k} \\
& \mathcal{F}_{g}(\lambda, \xi)=\frac{B_{2 g}}{2 g(2 g-2)} \lambda^{2-2 g}+\sum_{k \geq 1} f_{g, k} \lambda^{k}, \quad g \geq 2
\end{aligned}
$$

In writing the second term in the first line, we used the dilogarithm identity

$$
\mathrm{Li}_{2}(z)+\mathrm{Li}_{2}\left(\frac{1}{z}\right)=-\frac{\pi^{2}}{6}-\frac{1}{2} \log ^{2}(-z) .
$$

In the last line, $B_{2 g}$ are Bernoulli numbers. The coefficients $f_{g, k}$ are themselves non-trivial functions of the parameter $\xi$. For $g=0$, one finds, at the very first orders, 


$$
\begin{aligned}
& f_{0,3}=\pi^{2} \frac{1-3 \cosh (2 \xi)}{24 \cosh (\xi)} \\
& f_{0,4}=\pi^{4} \frac{-73+68 \cosh (2 \xi)+45 \cosh (4 \xi)}{2304 \cosh ^{2}(\xi)} \\
& f_{0,5}=\pi^{6} \frac{534-203 \cosh (2 \xi)-390 \cosh (4 \xi)-165 \cosh (6 \xi)}{30720 \cosh ^{3}(\xi)},
\end{aligned}
$$

while for $g=1,2$, one finds,

$$
\begin{aligned}
& f_{1,1}=\pi^{2} \frac{-1+3 \cosh (2 \xi)}{48 \cosh (\xi)} \\
& f_{1,2}=\pi^{4} \frac{127+4 \cosh (2 \xi)-27 \cosh (4 \xi)}{2304 \cosh ^{2}(\xi)} \\
& f_{1,3}=\pi^{6} \frac{-750-265 \cosh (2 \xi)+30 \cosh (4 \xi)+57 \cosh (6 \xi)}{18432 \cosh ^{3}(\xi)} \\
& f_{2,1}=\pi^{6} \frac{894+577 \cosh (2 \xi)+210 \cosh ^{2}(4 \xi)+15 \cosh (6 \xi)}{61440 \cosh ^{3}(\xi)}
\end{aligned}
$$

These results will be crucial to compare the asymptotic evaluation of the fermionic spectral traces to the predictions of [1].

\subsection{The Exact Planar Solution}

The $O(2)$ matrix model can be solved exactly in the planar limit $[17,42]$. However, it was noted in [19] that instead of using the specific results for the $O(2)$ case, it is more convenient to first consider the $O(n)$ model for arbitrary $n$, solve it with the powerful techniques of [18], and then take the limit $n \rightarrow 2$.

To proceed, we change variables $z=\mathrm{e}^{u}$ in the matrix integral (2.54), and we obtain

$$
Z(N, \hbar)=\frac{\mathrm{e}^{-\frac{\xi}{2 \pi \mathrm{g}^{2}} \lambda}}{N !} \int \frac{\mathrm{d}^{N} z}{(2 \pi)^{N}} \mathrm{e}^{-\frac{1}{\mathrm{~g}} \sum_{i=1}^{N}\left(V^{(0)}\left(z_{i}\right)+\mathcal{O}\left(\mathrm{g}^{2}\right)\right)} \frac{\prod_{i<j}\left(z_{i}-z_{j}\right)^{2}}{\prod_{i, j}\left(z_{i}+z_{j}\right)},
$$

where the classical potential (2.59), when written in terms of $z$, reads

$$
V^{(0)}(z)=-\frac{\log (z)}{2 \pi}+\frac{\operatorname{Im~Li}_{2}\left(\mathrm{i} z \mathrm{e}^{\xi}\right)+\operatorname{Im~Li}_{2}\left(\mathrm{i} z \mathrm{e}^{-\xi}\right)}{\pi^{2}} .
$$

To obtain the planar limit it is enough to consider the classical potential in (2.66). We assume that we can model the distribution of eigenvalues by a continuous function on a single connected compact support, i.e., we assume that we have a one-cut solution. This is a natural assumption, since the potential has a unique minimum at $u=0$ and it has a linearly confining behavior (2.61). We will take the cut along the segment $[a, b] \in \mathbb{R}_{+}$. Following the techniques of [18] (in the conventions of [19]) we introduce the auxiliary $G$-functions,

$$
\begin{gathered}
G^{(\nu)}(z)=-\mathrm{i}\left(\mathrm{e}^{\frac{\mathrm{i} \pi \nu}{2}} G_{+}^{(\nu)}(z)-\mathrm{e}^{-\frac{\mathrm{i} \pi \nu}{2}} G_{+}^{(\nu)}(-z)\right), \\
G^{(1-\nu)}(z)=-\left(\mathrm{e}^{\frac{\mathrm{i} \pi \nu}{2}} g_{+}(z) G_{+}^{(\nu)}(z)+\mathrm{e}^{-\frac{\mathrm{i} \pi \nu}{2}} g_{+}(-z) G_{+}^{(\nu)}(-z)\right),
\end{gathered}
$$


where

$$
\begin{gathered}
G_{+}^{(\nu)}(z)=\frac{-\mathrm{i} z}{\sqrt{z^{2}-a^{2}} \sqrt{z^{2}-b^{2}}} \frac{\vartheta_{4}(0) \vartheta_{1}\left(\pi \frac{v-\mathrm{i}(1-\nu) \mathrm{K}^{\prime}}{2 \mathrm{~K}}\right)}{\vartheta_{4}\left(\pi \frac{\mathrm{i}(1-\nu) \mathrm{K}^{\prime}}{2 \mathrm{~K}}\right) \vartheta_{1}\left(\pi \frac{v}{2 \mathrm{~K}}\right)} \mathrm{e}^{-\frac{\mathrm{i} \pi(1-\nu) v}{2 \mathrm{~K}}} \\
\text { with } z=a \operatorname{sn}(v) \\
g_{+}(z)=\frac{\sqrt{z^{2}-a^{2}} \sqrt{z^{2}-b^{2}}+\frac{z}{e} \sqrt{e^{2}-a^{2}} \sqrt{e^{2}-b^{2}}}{z^{2}-e^{2}} \\
\text { with } e=a \sin \left(\mathrm{i}(1-\nu) \mathrm{K}^{\prime}\right)
\end{gathered}
$$

Here, $\mathrm{K}$ and $\mathrm{K}^{\prime}$ are elliptic integrals of the first kind, and $\vartheta_{i}(u)$ are Jacobi theta functions. We follow the conventions of [43] for all the elliptic functions and integrals appearing in these formulae. The elliptic modulus $k$ and the nome $q\left(=\mathrm{e}^{\mathrm{i} \pi \tau}\right)$ are given by:

$$
k=\frac{a}{b}, \quad q=\mathrm{e}^{-\pi \frac{\mathrm{K}^{\prime}}{\mathrm{K}}} .
$$

In addition, the $\nu$ parameter is related to the $n$ of the $O(n)$ model by

$$
n=2 \cos (\pi \nu)
$$

so that $n \rightarrow 2$ corresponds to $\nu \rightarrow 0$.

Let us denote by $\mathcal{C}$ the closed contour encircling the cut at $[a, b]$ clockwise. The following equations, due to [18], allow to find the 't Hooft parameter $\lambda$ as a function of the endpoints of the cut $a, b$ :

$$
\begin{gathered}
0=\frac{1}{2 \cos \left(\frac{\pi(1-\nu)}{2}\right)} \oint_{\mathcal{C}} \frac{\mathrm{d} z}{2 \pi \mathrm{i}} \frac{\mathrm{d} V^{(0)}}{\mathrm{d} z} G^{(1-\nu)}(z), \\
\lambda=\frac{1}{2(1-\cos (\nu \pi)) \cos \left(\frac{\pi \nu}{2}\right)} \oint_{\mathcal{C}} \frac{\mathrm{d} z}{2 \pi \mathrm{i}} z \frac{\mathrm{d} V^{(0)}}{\mathrm{d} z} G^{(\nu)}(z) .
\end{gathered}
$$

The first equation is satisfied if we set $b=1 / a$, as expected from the symmetry $u_{i} \leftrightarrow-u_{i}$ of the matrix integral. So our elliptic modulus is given by $k=a^{2}$. The second equation leads, in the limit $\nu \rightarrow 0$, to the equation

$$
\lambda=\frac{f(\xi, a)}{\pi^{2}} .
$$

To determine the function $f(\xi, a)$, we note that the derivative $\partial f(\xi, a) / \partial \xi$ can be computed by deforming the contour and using the residue theorem. Indeed, we have

$$
\frac{\partial}{\partial \xi}\left(z \frac{\mathrm{d} V^{(0)}}{\mathrm{d} z}\right)=\frac{z}{2 \pi^{2} \mathrm{i}}\left(\frac{1}{z+\mathrm{ie}^{\xi}}-\frac{1}{z-\mathrm{ie}^{\xi}}-\frac{1}{z+\mathrm{ie}^{-\xi}}+\frac{1}{z-\mathrm{ie}^{-\xi}}\right) .
$$


After some calculations, one obtains

$$
\begin{aligned}
\frac{\partial}{\partial \xi} f(\xi, a)= & \frac{\mathrm{K}}{2 \pi \sqrt{\left(a^{2}+1\right)^{2}+4 a^{2} \sinh ^{2}(\xi)}} \\
& \left\{-\left(Z\left(\arcsin \frac{\mathrm{e}^{\xi}}{\sqrt{a^{2}+\mathrm{e}^{2 \xi}}}\right)-\frac{\mathrm{e}^{\xi} \sqrt{1+a^{2} \mathrm{e}^{2 \xi}}}{\sqrt{a^{2}+\mathrm{e}^{2 \xi}}}\right)^{2}\right. \\
& \left.+\left(Z\left(\arcsin \frac{\mathrm{e}^{-\xi}}{\sqrt{a^{2}+\mathrm{e}^{-2 \xi}}}\right)-\frac{\mathrm{e}^{-\xi} \sqrt{1+a^{2} \mathrm{e}^{-2 \xi}}}{\sqrt{a^{2}+\mathrm{e}^{-2 \xi}}}\right)^{2}+2 a^{2} \sinh (2 \xi)\right\},
\end{aligned}
$$

where $Z$ is the Jacobi Zeta function. The argument of the elliptic functions appearing in this and subsequent expressions is now given by the complementary modulus

$$
k_{1}=\sqrt{1-a^{4}}
$$

Since $f(\xi, a) \rightarrow 0$ when $\xi \rightarrow-\infty$, we can write

$$
f(\xi, a)=\int_{-\infty}^{\xi} \mathrm{d} \xi^{\prime} \frac{\partial}{\partial \xi^{\prime}} f\left(\xi^{\prime}, a\right) .
$$

A convenient expression for this integral is in terms of Jacobi theta functions with nome

$$
q_{1}=\mathrm{e}^{-\pi \frac{\mathrm{K}^{\prime}}{\mathrm{K}}}=\mathrm{e}^{\mathrm{i} \pi \tau_{1}}
$$

One finds,

$$
\begin{aligned}
f(\xi, a)= & \lim _{\Lambda \rightarrow \infty}\left[\frac{1}{4} \int_{\frac{\pi}{2 \mathrm{~K}} w(\xi)}^{\frac{\pi}{2 \mathrm{~K}} w(\Lambda)}\left(\frac{\vartheta_{2}^{\prime}}{\vartheta_{2}}(y)^{2}-\frac{\vartheta_{1}^{\prime}}{\vartheta_{1}}(y)^{2}\right) \mathrm{d} y\right. \\
& \left.+\frac{\mathrm{K}}{2 \pi}\left(\sqrt{\left(a^{2}+1\right)^{2}+4 a^{2} \sinh ^{2}(\xi)}-\sqrt{\left(a^{2}+1\right)^{2}+4 a^{2} \sinh ^{2}(\Lambda)}\right)\right]
\end{aligned}
$$

where

$$
w(\xi)=\mathrm{F}\left(\arcsin \frac{\mathrm{e}^{\xi}}{\sqrt{a^{2}+\mathrm{e}^{2 \xi}}}\right)
$$

and $\mathrm{F}$ is the incomplete elliptic integral of the first kind with modulus $k_{1}$. This equation determines the endpoints of the cut as functions of the ' $t$ Hooft parameter $\lambda$. The planar free energy is then determined by the equation $[18,19]$,

$$
\frac{\mathrm{d}^{2} \mathcal{F}_{0}}{\mathrm{~d} \lambda^{2}}=-2 \pi \frac{\mathrm{K}^{\prime}}{\mathrm{K}}=2 \log q_{1},
$$

up to two integration constants, which can be easily fixed by the weak coupling analysis of the previous section.

The exact planar solution makes it possible to explore the dependence of $\mathcal{F}_{0}$ on the full moduli space of $\lambda, \xi$. First of all, we can reproduce the perturbative results in (2.62), (2.64) by doing a small ' $t$ Hooft coupling expansion. When the 't Hooft parameter goes to zero, the cut collapses to the minimum 
of the potential. In the $z$-plane, the endpoint of the cut $a$ goes towards 1 , so we can expand in small $k_{1}=\sqrt{1-a^{4}}$. In this case, we can use the $q_{1}$-expansions of the theta functions in (2.82), and after some calculations we find,

$$
\begin{aligned}
\lambda= & \frac{1}{64 \pi^{2} \cosh (\xi)} k_{1}^{4}+\frac{1}{64 \pi^{2} \cosh (\xi)} k_{1}^{6}+\frac{115+119 \cosh (2 \xi)}{16384 \pi^{2} \cosh ^{3}(\xi)} k_{1}^{8} \\
& +\frac{51+55 \cosh (2 \xi)}{8192 \pi^{2} \cosh ^{3}(\xi)} k_{1}^{10}+\mathcal{O}\left(k_{1}^{12}\right) .
\end{aligned}
$$

Inverting this series and plugging it in (2.84), we obtain, after integrating twice,

$$
\begin{aligned}
\mathcal{F}_{0}(\lambda, \xi)= & c_{0}(\xi)+\left(c_{1}(\xi)-\frac{\xi}{2 \pi}\right) \lambda+\frac{\lambda^{2}}{2}\left(\log \frac{\pi^{2} \lambda \cosh \xi}{4}-\frac{3}{2}\right) \\
& +\frac{\pi^{2}(1-3 \cosh (2 \xi))}{24 \cosh (\xi)} \lambda^{3}+\frac{\pi^{4}(-73+68 \cosh (2 \xi)+45 \cosh (4 \xi))}{2304 \cosh ^{2}(\xi)} \lambda^{4} \\
& +\mathcal{O}\left(\lambda^{5}\right),
\end{aligned}
$$

where $c_{0,1}(\xi)$ are integration constants, and we added after integration the missing $-\xi \lambda /(2 \pi)$ from the prefactor of (2.66). This agrees with the perturbative expansion at genus zero from (2.62), (2.64).

One advantage of the exact solution is that we can also analyze the regime of strong 't Hooft coupling. For this, we do an $S$-transformation in (2.82) and express our formulae in terms of

$$
q=\mathrm{e}^{\mathrm{i} \pi \tau}=\mathrm{e}^{-\mathrm{i} \pi / \tau_{1}}
$$

which is the relevant variable for the large $\lambda$ expansion. We also do a shift in the integration variable to obtain:

$$
\begin{aligned}
& f(\xi, a) \\
& =\lim _{\Lambda \rightarrow \infty}\left\{-\frac{\tau}{4} \int_{\frac{\pi \tau}{2}(1-w(\xi) / \mathrm{K})}^{\frac{\pi \tau}{2}(1-w(\Lambda) / \mathrm{K})} \mathrm{d} y\left(\frac{\vartheta_{1}^{\prime}}{\vartheta_{1}}(y)-\frac{\vartheta_{4}^{\prime}}{\vartheta_{4}}(y)\right)\left(\frac{\vartheta_{1}^{\prime}}{\vartheta_{1}}(y)+\frac{\vartheta_{4}^{\prime}}{\vartheta_{4}}(y)+\frac{4 \mathrm{i} y}{\pi \tau}\right)\right. \\
& \left.\quad+\frac{\mathrm{K}}{2 \pi}\left(\sqrt{\left(a^{2}+1\right)^{2}+4 a^{2} \sinh ^{2}(\xi)}-\sqrt{\left(a^{2}+1\right)^{2}+4 a^{2} \sinh ^{2}(\Lambda)}\right)\right\},
\end{aligned}
$$

where the elliptic integrals are still evaluated at $k_{1}$. As we did for the weak coupling expansion, we expand the integrand in small $q$ and integrate. After some calculations, we obtain

$$
\begin{aligned}
\lambda= & \frac{1}{8 \pi^{3}} \log ^{2} \frac{k}{4}-\frac{1}{12 \pi}-\frac{\xi^{2}}{2 \pi^{3}}+\frac{1}{\pi^{3}} \cosh (2 \xi)\left(1-\log \frac{k}{4}\right)\left(\frac{k}{4}\right) \\
& +\frac{1}{4 \pi^{3}}\left\{4\left(1-\log \frac{k}{4}\right)+3 \cosh (4 \xi)\left(-1+2 \log \frac{k}{4}\right)\right\}\left(\frac{k}{4}\right)^{2}+\mathcal{O}\left(k^{3}\right) .
\end{aligned}
$$


where we remind that $k=a^{2}$. This can be inverted to yield the series,

$$
\frac{k}{4}=\mathrm{e}^{-2 \pi \sqrt{2 \hat{\lambda}}}+\left(4+\frac{\sqrt{2}}{\pi \sqrt{\hat{\lambda}}}\right) \cosh (2 \xi) \mathrm{e}^{-4 \pi \sqrt{2 \hat{\lambda}}}+\mathcal{O}\left(\mathrm{e}^{-6 \pi \sqrt{2 \hat{\lambda}}}\right),
$$

where we use the shorthand notation

$$
\hat{\lambda}=\pi \lambda+\frac{1}{12}+\frac{\xi^{2}}{2 \pi^{2}}
$$

Again, by (2.84), we finally obtain,

$$
\begin{aligned}
\mathcal{F}_{0}(\lambda, \xi)= & -\frac{\sqrt{2} \hat{\lambda}^{3 / 2}}{3 \pi}+\tilde{c}_{0}(\xi)+\left(\tilde{c}_{1}(\xi)-\frac{\xi}{2 \pi}\right) \lambda-\frac{\cosh (2 \xi)}{4 \pi^{4}} \mathrm{e}^{-2 \pi \sqrt{2 \hat{\lambda}}} \\
& -\frac{1}{32 \pi^{5}}\left\{\left(8 \pi+\frac{4}{\sqrt{2 \hat{\lambda}}}\right)+\left(\pi+\frac{4}{\sqrt{2 \hat{\lambda}}}\right) \cosh (4 \xi)\right\} \mathrm{e}^{-4 \pi \sqrt{2 \hat{\lambda}}} \\
& +\mathcal{O}\left(\mathrm{e}^{-6 \pi \sqrt{2 \hat{\lambda}}}\right)
\end{aligned}
$$

where $\tilde{c}_{0,1}(\xi)$ are integration constants that can be fixed in principle from the weak coupling behavior. As anticipated in [1], the strong coupling expansion of the free energy displays the $3 / 2$ scaling typical of theories of M2 branes [44], and the coefficient of the leading term agrees with the general formula for local del Pezzo Calabi-Yau's found in [1]. The expansion (2.92) is very similar to the expansion of the planar free energy of ABJ(M) theory presented in [24]. As we will see in the next section, one can in fact recover the result for $\operatorname{ABJ}(\mathrm{M})$ theory from (2.92). Let us also note that, in the case $\xi=0$, the formulae above simplify considerably, and one can write the periods in terms of indefinite integrals of theta functions,

$$
\begin{gathered}
\lambda=-\frac{1}{2 \pi \mathrm{i}} \int \mathrm{d} \tau_{1} \vartheta_{2}\left(2 \tau_{1}\right)^{4} \vartheta_{3}\left(2 \tau_{1}\right)^{2}, \\
\frac{\mathrm{d} \mathcal{F}_{0}}{\mathrm{~d} \lambda}=-\int \mathrm{d} \tau_{1} \tau_{1} \vartheta_{2}\left(2 \tau_{1}\right)^{4} \vartheta_{3}\left(2 \tau_{1}\right)^{2}+c,
\end{gathered}
$$

where $c$ is again an integration constant. These integrals can be performed to obtain an expression which is useful for strong coupling expansions, namely,

$$
\begin{aligned}
\lambda= & \frac{1}{4 \pi^{4}} G_{3,3}^{3,2}\left(\frac{4 k}{(k+1)^{2}} \mid \begin{array}{c}
\frac{1}{2}, \frac{1}{2}, 1 \\
0,0,0
\end{array}\right)+C_{1}, \\
\frac{\partial \mathcal{F}_{0}}{\partial \lambda}= & \frac{4 k}{16 \pi(k+1)^{2}}{ }_{4} F_{3}\left(1,1, \frac{3}{2}, \frac{3}{2} ; 2,2,2 ; \frac{4 k}{(k+1)^{2}}\right) \\
& +\frac{1}{4 \pi} \log \left(-\frac{4 k}{(k+1)^{2}}\right)+C_{2} .
\end{aligned}
$$

Another ingredient of the planar solution which can be computed exactly is the density of eigenvalues. Let us first consider the resolvent of the $O(2)$ matrix model (2.66), defined as

$$
\omega(p)=\frac{1}{N}\left\langle\operatorname{Tr} \frac{1}{p-M}\right\rangle,
$$


where $M$ is the matrix with eigenvalues $z_{i}, i=1, \ldots, N$, and the bracket denotes the normalized vev. We can split this function into its even and odd parts with respect to $p$,

$$
\omega(p)=\omega_{+}(p)+\omega_{-}(p) .
$$

The planar limit of the even part can be computed using the formula [17]

$$
\omega_{+}^{0}(p)=-\frac{1}{2 \lambda} \oint_{\mathcal{C}} \frac{\mathrm{d} w}{2 \pi \mathrm{i}} \frac{V^{\prime}(w) w}{p^{2}-w^{2}} \frac{\sqrt{\left(p^{2}-a^{2}\right)\left(p^{2}-\frac{1}{a^{2}}\right)}}{\sqrt{\left(w^{2}-a^{2}\right)\left(w^{2}-\frac{1}{a^{2}}\right)}},
$$

where $V(z)$ is the planar potential (2.67), and $\mathcal{C}$ is a contour around the cut $[a, 1 / a]$ anti-clockwise. We find,

$$
\begin{aligned}
\omega_{+}^{0}(p)= & \frac{a \sqrt{\left(p^{2}-a^{2}\right)\left(p^{2}-\frac{1}{a^{2}}\right)}}{4 \pi^{2} \mathrm{i} p^{2} \lambda}\left\{\Pi\left(\frac{a^{2}}{p^{2}}, \arcsin \frac{\mathrm{ie}^{\xi}}{a} \mid a^{4}\right)\right. \\
& \left.+\Pi\left(\frac{a^{2}}{p^{2}}, \arcsin \frac{\mathrm{ie}^{-\xi}}{a} \mid a^{4}\right)-L(p, a)\right\} \\
& +\frac{1}{4 \pi^{2} p \lambda}\left(\arctan \frac{\mathrm{e}^{\xi}}{p}-\arctan \mathrm{e}^{\xi} p\right),
\end{aligned}
$$

where $\Pi$ is the elliptic integral of the third kind, and

$$
\begin{aligned}
L(p, a) & =\lim _{\Lambda \rightarrow \infty} \Pi\left(\frac{a^{2}}{p^{2}}, \mathrm{i} \Lambda \mid a^{4}\right) \\
& =\frac{-\mathrm{i} p^{2}}{a^{2}\left(a^{2}-p^{2}\right)}\left[\Pi\left(1-\frac{p^{2}}{a^{2}} \mid 1-\frac{1}{a^{4}}\right)-\mathrm{K}^{\prime}\left(\frac{1}{a^{4}}\right)\right] .
\end{aligned}
$$

When $z \in[a, 1 / a]$, the eigenvalue density is given by the discontinuity equation

$$
\begin{aligned}
\rho(z)= & \frac{1}{\mathrm{i} \pi}\left(\omega_{+}^{0}(z+\mathrm{i} 0)-\omega_{+}^{0}(z-\mathrm{i} 0)\right) \\
= & \frac{a \sqrt{\left(z^{2}-a^{2}\right)\left(\frac{1}{a^{2}}-z^{2}\right)}}{2 \pi^{3} \mathrm{i} z^{2} \lambda} \times\left\{\Pi\left(\frac{a^{2}}{z^{2}}, \arcsin \frac{\mathrm{ie}^{\xi}}{a} \mid a^{4}\right)\right. \\
& \left.+\Pi\left(\frac{a^{2}}{z^{2}}, \arcsin \frac{\mathrm{ie}^{-\xi}}{a} \mid a^{4}\right)-L(z, a)\right\} .
\end{aligned}
$$

This expression can be checked by doing a numerical simulation of 300 eigenvalues relaxed into a configuration which approximately minimizes the effective action of the matrix model, as in [45]. The results are shown in Fig. 2, after going back to the initial $u$ variable, so that $\rho(u)=\rho(z(u)) \mathrm{d} z / \mathrm{d} u$, with $z(u)=\mathrm{e}^{u}$.

\subsection{Relation to the ABJ(M) Matrix Model}

In [46], a matrix model computing the partition function of $\mathrm{ABJ}(\mathrm{M})$ theory $[47,48]$ on $\mathbb{S}^{3}$ was derived, using localization. This model turns out to 

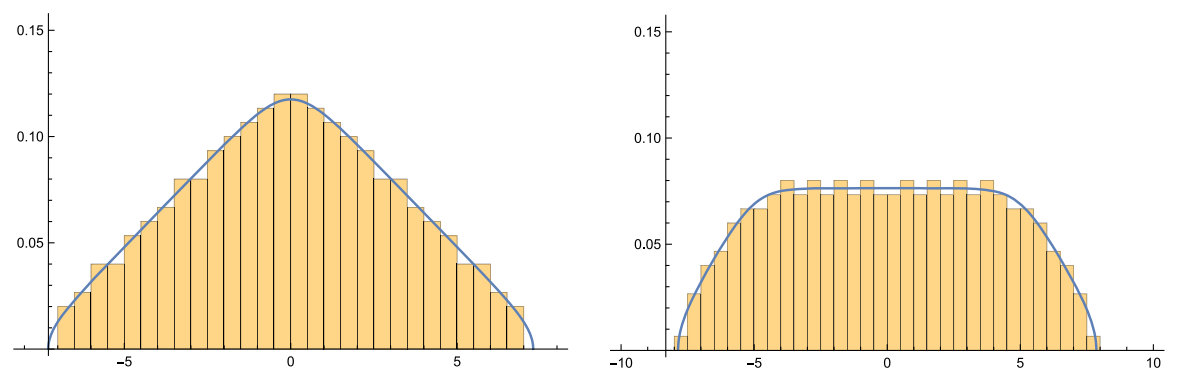

Figure 2. Left Eigenvalue density $\rho(u)$ for $\lambda=1, \xi=0$ against a histogram showing the numerical density of $N=300$ relaxed eigenvalues. Right Same plot with $\lambda=3 / 4$ and $\xi=5$

be closely related to the topological string on local $\mathbb{P}^{1} \times \mathbb{P}^{1}$. In the case of ABJM theory, this was first noted and exploited in $[6,24]$ in the context of the ' $t$ Hooft expansion, and then in $[9,10]$ for the M-theory expansion (which involves as well the refined topological string in the NS limit). The generalization to ABJ theory was done in [23,49]. Since the matrix model $(2.31)$ gives a non-perturbative completion of the partition function on this geometry, it is natural to wonder whether it is related to the ABJ(M) matrix model. In fact, one can recover the $\mathrm{ABJ}(\mathrm{M})$ matrix model from (2.31) provided one considers complex values of the parameter $m_{\mathbb{F}_{0}}$. Note that the operator (2.10) is no longer self-adjoint in this case, and, in addition, one has to be careful with the resulting multi-valued structure, since the integral kernel depends on the logarithm of $m_{\mathbb{F}_{0}}$.

Let us then set

$$
\log m_{\mathbb{F}_{0}}=\mathrm{i} \hbar-2 \pi \mathrm{i} M, \quad M \in \mathbb{Z}_{\geq 0} .
$$

Here, the integer $M$ will be identified with the difference between the ranks of the two Chern-Simons theories in ABJ theory [48]. This relationship is the one suggested by the explicit results of $[23,49]$. In these papers, the grand potential of $\mathrm{ABJ}(\mathrm{M})$ theory is written in terms of the topological string on local $\mathbb{F}_{0}$. If we now use the explicit expression (2.20) for the integral kernel, we find

$$
\begin{aligned}
\rho_{\mathbb{F}_{0}}= & \mathrm{e}^{-\mathrm{i} \hbar / 4+\mathrm{i} \pi M / 2} \mathrm{e}^{\sqrt{\pi \hbar} \mathrm{q} / 2} \frac{\Phi \sqrt{\hbar / \pi}\left(\mathrm{q}+\frac{\mathrm{i}}{2} M \sqrt{\pi / \hbar}\right)}{\Phi \sqrt{\hbar / \pi}\left(\mathrm{q}-\frac{\mathrm{i}}{2} M \sqrt{\pi / \hbar}\right)} \frac{1}{2 \cosh (\sqrt{\pi \hbar} \mathrm{p})} \\
& \times \frac{\Phi \sqrt{\hbar / \pi}\left(\mathrm{q}-\frac{\mathrm{i}}{2} M \sqrt{\pi / \hbar}+\frac{\mathrm{i}}{2} \sqrt{\hbar / \pi}\right)}{\Phi \sqrt{\hbar / \pi}\left(\mathrm{q}+\frac{\mathrm{i}}{2} M \sqrt{\pi / \hbar}-\frac{\mathrm{i}}{2} \sqrt{\hbar / \pi}\right)} \mathrm{e}^{\sqrt{\pi \hbar} \mathrm{q} / 2} .
\end{aligned}
$$

Due to the form of the arguments, we can use the functional equations for the quantum dilogarithm, (A.9a) and (A.9b), and we obtain 


$$
\begin{aligned}
\rho_{\mathbb{F}_{0}}= & \mathrm{e}^{-\mathrm{i} \hbar / 4+\mathrm{i} \pi M / 2} \mathrm{e}^{\sqrt{\pi \hbar} \mathrm{q} / 2}\left(\prod_{s=\frac{-M+1}{2}}^{\frac{M-1}{2}} \frac{1}{1+\mathrm{e}^{2 \pi(\mathrm{q} \sqrt{\pi / \hbar}+\mathrm{i} \pi s / \hbar)}}\right) \frac{1}{2 \cosh (\sqrt{\pi \hbar} \mathrm{p})} \\
& \times\left(\prod_{s=\frac{-M+1}{2}}^{\frac{M-1}{2}}\left(1+\mathrm{e}^{2 \pi(\mathrm{q} \sqrt{\pi / \hbar}+\mathrm{i} \pi s / \hbar+\mathrm{i} / 2)}\right)\right) \frac{1}{1+(-1)^{M} \mathrm{e}^{2 \sqrt{\pi \hbar} \mathrm{q}}} \mathrm{e}^{\sqrt{\pi \hbar} \mathrm{q} / 2} .
\end{aligned}
$$

To make contact with $\mathrm{ABJ}(\mathrm{M})$ theory, let us define

$$
k=\frac{\hbar}{\pi}
$$

and let us introduce the variables

$$
\mathrm{u}=2 \pi \sqrt{k} \mathbf{q}, \quad \mathbf{v}=2 \pi \sqrt{k} \mathrm{p},
$$

so that $[\mathrm{u}, \mathrm{v}]=2 \pi \mathrm{i} k$. In these new variables, and after a similarity transformation, we find,

$$
A \rho_{\mathbb{F}_{0}} A^{-1}=\mathrm{e}^{-\mathrm{i} \pi k / 4+\mathrm{i} \pi M / 2} \rho_{\mathrm{ABJ}(\mathrm{M})},
$$

where

$$
\rho_{\mathrm{ABJ}(\mathrm{M})}=\frac{1}{2 \cosh (\mathrm{v} / 2)} \frac{1}{\mathrm{e}^{\frac{\mathrm{u}}{2}}+(-1)^{M} \mathrm{e}^{-\frac{\mathrm{u}}{2}}} \prod_{s=\frac{-M+1}{2}}^{\frac{M-1}{2}} \tanh \left(\frac{\mathrm{u}+2 \pi \mathrm{i} s}{2 k}\right)
$$

is, up to a similarity transformation, the operator appearing in the Fermi gas formulation of ABJM theory [6] and of ABJ theory [21-23]. Since the phase appearing in (2.107) is the same one appearing in the relation between $\mathbb{F}_{0}$ and $\mathbb{F}_{2}$, we also conclude that,

$$
\rho_{\mathbb{F}_{2}}=\rho_{\mathrm{ABJ}(\mathrm{M})},
$$

up to a combination of unitary and similarity transformations. The dictionary between the parameters is (2.105) and

$$
m_{\mathbb{F}_{2}}=2 \cos \left(\frac{\pi k}{2}-\pi M\right) .
$$

In particular, the spectral traces of the kernel of the ABJ(M) matrix model can be obtained from the traces of the $\mathbb{F}_{2}$ operator. This can be easily tested for ABJM theory, in the case $k=2, M=0$, using the expressions (2.48). The relevant value of the mass parameter is $m_{\mathbb{F}_{2}}=-2$, which is a branch point for the functions in (2.48). However, the traces at this point are well-defined and one finds the correct values [7]

$$
\left.\operatorname{Tr} \rho_{\mathrm{ABJM}}\right|_{k=2}=\frac{1}{8},\left.\quad \operatorname{Tr} \rho_{\mathrm{ABJM}}^{2}\right|_{k=2}=\frac{1}{64}-\frac{1}{16 \pi^{2}} .
$$

We also find that, when $k=2$ and $M=1$, which is the maximally supersymmetric ABJ theory, the theory is equivalent (at the level of spectral traces) to the maximally supersymmetric case of local $\mathbb{F}_{2}$ with $m_{\mathbb{F}_{2}}=2$, or, equivalently, of local $\mathbb{F}_{0}$ with $m_{\mathbb{F}_{0}}=1$. 
The exact results for the planar solution found in the previous section can be used to rederive the exact planar solution of the ABJ $(\mathrm{M})$ matrix model, first obtained in $[24,50]$. Indeed, due to $(2.102)$, the exact planar free energy of $\mathrm{ABJ}(\mathrm{M})$ theory can be obtained from the general formulae obtained above by setting

$$
\xi=\frac{\mathrm{i} \pi}{2}-\frac{\mathrm{i} \pi^{2} M}{\hbar} .
$$

As a check, note that the shifted variable (2.91) becomes

$$
\hat{\lambda}=\pi \lambda-\frac{1}{2}\left(B^{2}-\frac{1}{4}\right)-\frac{1}{24}
$$

where

$$
B=\frac{1}{2}-\frac{M}{k}
$$

has to be identified as the $B$ field of ABJ theory. The shift (2.113) is precisely the one found in [24]. In addition, the strong coupling expansion (2.92) becomes,

$$
\begin{aligned}
\mathcal{F}_{0}(\lambda, \beta)= & -\frac{\sqrt{2} \hat{\lambda}^{3 / 2}}{3 \pi}+\tilde{c}_{0}+\left(\tilde{c}_{1}-\frac{\mathrm{i}}{4}\right) \lambda+\frac{\beta+\beta^{-1}}{8 \pi^{4}} \mathrm{e}^{-2 \pi \sqrt{2 \hat{\lambda}}} \\
& -\frac{1}{4 \pi^{4}}\left\{\frac{1}{16}\left(\beta^{2}+16+\beta^{-2}\right)+\frac{1}{4 \pi \sqrt{2 \hat{\lambda}}}\left(\beta+\beta^{-1}\right)^{2}\right\} \mathrm{e}^{-4 \pi \sqrt{2 \hat{\lambda}}} \\
& +\mathcal{O}\left(\mathrm{e}^{-6 \pi \sqrt{2 \hat{\lambda}}}\right)
\end{aligned}
$$

where

$$
\beta=\mathrm{e}^{-2 \pi \mathrm{i} M / k} \text {. }
$$

The function in (2.115) is precisely $-1 /\left(4 \pi^{4}\right)$ times the planar free energy $F_{0}^{\mathrm{ABJ}}$ obtained in [24]. This overall factor is due to our different conventions for the string coupling constant.

It should be noted, however, that the perturbative and weak coupling expansion worked out for the matrix model (2.54) can not be used for ABJM theory in the form presented above. For ABJM theory, $M=0$, so that $\xi=$ $\mathrm{i} \pi / 2$, and the expansions (2.64), (2.65) and (2.86) diverge. This is not a problem of our exact solution, but rather a breakdown of the Gaussian approximation. The reason is that, when considering the particular limit of ABJM theory, the "classical" potential (2.59) is no longer a perturbed Gaussian, since it is exactly given by the r.h.s. of (2.61), and, in particular, it is not smooth at $u=0$. One can, however, still obtain the correct weak coupling expansion from the exact planar solution, and one obtains,

$$
\lambda=\frac{1}{8 \pi^{2}} k_{1}^{2}+\frac{1}{16 \pi^{2}} k_{1}^{4}+\frac{65}{1536 \pi^{2}} k_{1}^{6}+\mathcal{O}\left(k_{1}^{8}\right)
$$


where $k_{1}$ is given by (2.79), as well as

$$
\begin{aligned}
\mathcal{F}_{0}(\lambda)= & c_{0}+c_{1} \lambda+\left(\log \left(\frac{\pi^{2} \lambda}{2}\right)-\frac{3}{2}\right) \lambda^{2}-\frac{\pi^{4}}{9} \lambda^{4} \\
& +\frac{283 \pi^{8}}{5400} \lambda^{6}-\frac{961 \pi^{12}}{19845} \lambda^{8}+\mathcal{O}\left(\lambda^{10}\right)
\end{aligned}
$$

which is precisely (up to an overall factor $-1 /\left(4 \pi^{4}\right)$ ) the expression found in [24]. In addition, one finds the relations

$$
\begin{aligned}
\lambda & =-\frac{1}{2 \pi \mathrm{i}} \int \mathrm{d} \tau_{1} \vartheta_{3}\left(2 \tau_{1}\right)^{4} \vartheta_{2}\left(2 \tau_{1}\right)^{2}, \\
\frac{\mathrm{d} \mathcal{F}_{0}}{\mathrm{~d} \lambda} & =-\int \mathrm{d} \tau_{1} \tau_{1} \vartheta_{3}\left(2 \tau_{1}\right)^{4} \vartheta_{2}\left(2 \tau_{1}\right)^{2}+\tilde{c},
\end{aligned}
$$

which are obtained from (2.93) by exchanging $\vartheta_{2}$ with $\vartheta_{3}$. This can be also integrated explicitly, as in (2.94), and the result is in precise agreement with the result of [24].

\section{Comparing the Matrix Model to the Topological String}

\subsection{Predictions from the Spectral Theory/Mirror Symmetry Correspondence}

The conjecture of [1] gives a very precise prediction for the ' $t$ Hooft expansion (1.2) of the fermionic traces of the trace class operators obtained by quantizing mirror curves. We will now summarize some of the results of [1], specialized to the case of interest, namely local $\mathbb{P}^{1} \times \mathbb{P}^{1}$ (see also $[13,14]$ for other summaries of the main results of [1]). According to the conjecture of [1], the basic quantity determining the spectral properties of the operator $\rho_{\mathbb{F}_{0}}$ is the modified grand potential $J\left(\mu, m_{\mathbb{F}_{0}}, \hbar\right)$. This function depends on the "chemical potential" $\mu$, which is related to the "fugacity" $\kappa$ entering (2.26) as

$$
\kappa=\mathrm{e}^{\mu}
$$

as well as on the mass parameter $m_{\mathbb{F}_{0}}$ appearing in (2.10). The modified grand potential is determined by the enumerative geometry of the CY. We first need a dictionary between the parameters $\mu, m_{\mathbb{F}_{0}}$, and the parameters appearing in the enumerative geometry of local $\mathbb{P}^{1} \times \mathbb{P}^{1}$. This CY has a "diagonal" Kähler parameter $T$, which is related to $\mu$ by

$$
T=2 \mu_{\text {eff }} .
$$

Here, the "effective" $\mu$ parameter is determined by the so-called quantum mirror map of [4] (see [1] for the notation),

$$
\mu_{\mathrm{eff}}=\mu-\frac{1}{2} \sum_{\ell \geq 1} \widehat{a}_{\ell}(\hbar) \mathrm{e}^{-2 \ell \mu} .
$$

In this paper we will not need the explicit expression of this quantum mirror map, since it is not relevant in the 't Hooft limit we will focus on. In addition, 
there is a Kähler parameter $T_{m}$ associated to the mass parameter $m_{\mathbb{F}_{0}}$. Geometrically it measures, roughly speaking, the difference in sizes between the two spheres in local $\mathbb{P}^{1} \times \mathbb{P}^{1}$, and one has the relation

$$
T_{m}=-\log m_{\mathbb{F}_{0}} .
$$

The modified grand potential has the form,

$$
J\left(\mu, m_{\mathbb{F}_{0}}, \hbar\right)=J^{(\mathrm{p})}\left(\mu_{\mathrm{eff}}, m_{\mathbb{F}_{0}}, \hbar\right)+J_{\mathrm{M} 2}\left(\mu_{\mathrm{eff}}, m_{\mathbb{F}_{0}}, \hbar\right)+J_{\mathrm{WS}}\left(\mu_{\mathrm{eff}}, m_{\mathbb{F}_{0}}, \hbar\right) .
$$

Here, $J^{(\mathrm{p})}\left(\mu, m_{\mathbb{F}_{0}}, \hbar\right)$ is the perturbative part, which is a cubic polynomial in $\mu$ :

$$
J^{(\mathrm{p})}\left(\mu, m_{\mathbb{F}_{0}}, \hbar\right)=\frac{2}{3 \pi \hbar} \mu^{3}-\frac{\log m_{\mathbb{F}_{0}}}{2 \pi \hbar} \mu^{2}+\left(\frac{\pi}{3 \hbar}-\frac{\hbar}{12 \pi}\right) \mu+A\left(m_{\mathbb{F}_{0}}, \hbar\right) .
$$

When $m_{\mathbb{F}_{0}}=1$ one recovers the expression presented in [1]. When $m_{\mathbb{F}_{0}} \neq 1$, the part which depends on $\mu$ can be obtained in a relatively straightforward way by working out the classical grand potential $[16,51]$, or using the result for local $\mathbb{F}_{2}$ and the dictionary between this model and local $\mathbb{F}_{0}$. A precise expression for the function $A\left(m_{\mathbb{F}_{0}}, \hbar\right)$ has been obtained by Y. Hatsuda (Unpublished.). His expression exploits the relationship between ABJ theory and topological string theory on local $\mathbb{P}^{1} \times \mathbb{P}^{1}$ discussed in Sect. 2.5. It is given by,

$$
A\left(m_{\mathbb{F}_{0}}, \hbar\right)=\frac{\log ^{3} m_{\mathbb{F}_{0}}}{48 \pi \hbar}-\frac{\log m_{\mathbb{F}_{0}}}{4}\left(\frac{\pi}{3 \hbar}-\frac{\hbar}{12 \pi}\right)+A_{\mathrm{c}}\left(\frac{\hbar}{\pi}\right)-F_{\mathrm{CS}}\left(\frac{\hbar}{\pi}, M\right) .
$$

Let us spell out the details of this formula. The function $A_{\mathrm{c}}(k)$ was introduced in [6] in the Fermi gas approach to ABJM theory. It was determined explicitly in [51] and further simplified in [52]. It reads,

$$
A_{\mathrm{c}}(k)=\frac{2 \zeta(3)}{\pi^{2} k}\left(1-\frac{k^{3}}{16}\right)+\frac{k^{2}}{\pi^{2}} \int_{0}^{\infty} \frac{x}{\mathrm{e}^{k x}-1} \log \left(1-\mathrm{e}^{-2 x}\right) \mathrm{d} x .
$$

In $(3.7), F_{\mathrm{CS}}(k, M)$ is an analytic continuation of the Chern-Simons free energy on the three-sphere for gauge group $U(M)$ and level $k$,

$$
F_{\mathrm{CS}}(k, M)=\log Z_{\mathrm{CS}}(k, M),
$$

where $M$ is related to the parameters of our problem as

$$
M=\frac{\hbar+\mathrm{i} \log m_{\mathbb{F}_{0}}}{2 \pi} .
$$

Note that this is precisely the relation (2.102) used in Sect. 2.5. As it is wellknown, the Chern-Simons partition function for integer $M$ is given by [53]

$$
Z_{\mathrm{CS}}(k, M)=k^{-M / 2} \prod_{j=1}^{M}\left(2 \sin \frac{\pi j}{k}\right)^{M-j},
$$


but in view of (3.10) we have to extend it to arbitrary complex $M$. This can be done in various equivalent ways $[54,55]$, but in this paper we will not need the precise form of this extension.

The "membrane" part of the potential $J_{\mathrm{M} 2}\left(\mu_{\mathrm{eff}}, m_{\mathrm{F}_{0}}, \hbar\right)$ appearing in (3.5) will not be relevant for our purposes. It is fully determined by the refined BPS invariants of the topological string in this CY background, see $[1,13]$ for details. Finally, the worldsheet part of the modified grand potential is

$$
J_{\mathrm{WS}}\left(\mu_{\mathrm{eff}}, m_{\mathbb{F}_{0}}, \hbar\right)=\sum_{g \geq 0} \sum_{v=1}^{\infty} \sum_{\mathbf{d}} n_{g}^{\mathbf{d}} \frac{1}{v}\left(2 \sin \frac{2 \pi^{2} v}{\hbar}\right)^{2 g-2} \mathrm{e}^{-\frac{2 \pi}{\hbar} v \mathbf{d} \cdot \mathbf{T}},
$$

where

$$
\mathbf{T}=\left(T, T_{m}\right),
$$

$n_{g}^{\mathbf{d}}$ are the Gopakumar-Vafa invariants [56] of local $\mathbb{P}^{1} \times \mathbb{P}^{1}$, and $\mathbf{d}=\left(d_{1}, d_{2}\right)$ are two non-negative integers.

One of the consequences of the conjecture of [1] is that the fermionic spectral traces $Z_{\mathbb{F}_{0}}(N, \hbar)$ can be obtained as integral transforms of the modified grand potential,

$$
Z_{\mathbb{F}_{0}}(N, \hbar)=\frac{1}{2 \pi \mathrm{i}} \int_{\mathcal{C}} \mathrm{e}^{J\left(\mu, m_{\mathbb{F}_{0}}, \hbar\right)-N \mu} \mathrm{d} \mu,
$$

where the contour $\mathcal{C}$ goes from $\mathrm{e}^{-\mathrm{i} \pi / 3} \infty$ to $\mathrm{e}^{\mathrm{i} \pi / 3} \infty$ (so that the integral is absolutely convergent). The formula (3.14) leads to a precise prediction for the 't Hooft limit of the fermionic spectral traces. Note that, to keep the dependence on both Kähler parameters, we have to take a limit in which $\log m_{\mathbb{F}_{0}}$ scales with $\hbar$, as we required in (2.51). We then consider the 't Hooft limit of $J\left(\mu, m_{\mathbb{F}_{0}}, \hbar\right)$, in which

$$
\mu \rightarrow \infty, \quad m_{\mathbb{F}_{0}} \rightarrow \infty, \quad \hbar \rightarrow \infty,
$$

and

$$
\frac{\mu}{\hbar}=\zeta \quad \text { fixed, } \quad \frac{\pi}{2 \hbar} \log m_{\mathbb{F}_{0}}=\xi \quad \text { fixed. }
$$

The parameter $\xi$ was introduced in (2.52). We will express often the results in terms of

$$
m=\mathrm{e}^{4 \xi},
$$

which corresponds to the mass parameter appearing in the standard topological string free energies. Indeed, with this definition, one has that

$$
-\log (m)=\frac{2 \pi}{\hbar} T_{m} .
$$

In the ' $t$ Hooft limit, the membrane part of the grand potential in (3.5) goes to zero, and $\mu_{\mathrm{eff}} \rightarrow \mu$. The remaining ingredients have non-trivial 't Hooft-like expansions. The expansion of $A\left(m_{\mathbb{F}_{0}}, \hbar\right)$ can be easily worked out. The function $A_{\mathrm{c}}(k)$ has the large $k$ expansion [51]: 


$$
\begin{aligned}
A_{\mathrm{c}}(k)= & -\frac{k^{2}}{8 \pi^{2}} \zeta(3)+\frac{1}{2} \log (2)+2 \zeta^{\prime}(-1)+\frac{1}{6} \log \left(\frac{\pi}{2 k}\right) \\
& +\sum_{g \geq 2}\left(\frac{2 \pi}{k}\right)^{2 g-2} 4^{g}(-1)^{g-1} c_{g},
\end{aligned}
$$

where

$$
c_{g}=\frac{B_{2 g} B_{2 g-2}}{(4 g)(2 g-2)(2 g-2) !} .
$$

On the other hand, in the limit we are considering, $M \rightarrow \infty$, but

$$
\frac{2 \pi^{2} \mathrm{i}}{\hbar} M=\pi \mathrm{i}-2 \xi
$$

is fixed. This is the standard 't Hooft expansion of $F_{\mathrm{CS}}(\hbar / \pi, M)$, worked out at all genus in [26], and with 't Hooft parameter (3.21). One then finds an expansion of the form,

$$
A\left(m_{\mathbb{F}_{0}}, \hbar\right)=\sum_{g \geq 0} A_{g}(\xi) \hbar^{2-2 g},
$$

where $A_{1}(\xi)$ includes as well a logarithmic dependence on $\hbar$, and

$$
\begin{aligned}
& A_{0}(\xi)=\frac{\zeta(3)-2 \mathrm{Li}_{3}\left(-\mathrm{e}^{2 \xi}\right)}{8 \pi^{4}} \\
& A_{1}(\xi)=-\frac{\xi}{6}+\frac{1}{12} \log \left(16 \pi^{2} \cosh \xi\right)-\frac{1}{12} \log \hbar+\zeta^{\prime}(-1) \\
& A_{g}(\xi)=\left(2 \pi^{2}\right)^{2 g-2}(-1)^{g-1}\left\{\left(4^{g}-2\right) c_{g}-\frac{B_{2 g}}{2 g(2 g-2) !} \operatorname{Li}_{3-2 g}\left(-\mathrm{e}^{2 \xi}\right)\right\}
\end{aligned}
$$

It follows from this expression that

$$
A_{g}(0)=2(-1)^{g-1}\left(4 \pi^{2}\right)^{2 g-2} c_{g}\left(3-2^{3-2 g}\right), \quad g \geq 2,
$$

in agreement with the result presented in [1] for $m_{\mathbb{F}_{0}}=1$,

$$
A\left(m_{\mathbb{F}_{0}}=1, \hbar\right)=\frac{3}{2} A_{\mathrm{c}}\left(\frac{\hbar}{\pi}\right)-A_{\mathrm{c}}\left(\frac{2 \hbar}{\pi}\right) .
$$

One concludes that, in the 't Hooft limit (3.15), the modified grand potential has the asymptotic expansion,

$$
J^{\text {t Hooft }}(\zeta, \xi, \hbar)=\sum_{g=0}^{\infty} J_{g}(\zeta, \xi) \hbar^{2-2 g},
$$

where

$$
\begin{aligned}
J_{0}(\zeta, \xi) & =\frac{2}{3 \pi} \zeta^{3}-\frac{\log m}{4 \pi^{2}} \zeta^{2}-\frac{1}{12 \pi} \zeta+A_{0}(\xi)+\frac{1}{16 \pi^{4}} F_{0}^{\text {inst }}(t, m), \\
J_{1}(\zeta, \xi) & =\frac{\pi}{3} \zeta+A_{1}(\xi)+F_{1}^{\text {inst }}(t, m), \\
J_{g}(\zeta, \xi) & =A_{g}(\xi)+\left(4 \pi^{2}\right)^{2 g-2} F_{g}^{\text {inst }}(t, m), \quad g \geq 2 .
\end{aligned}
$$


Here, we have introduced the variable

$$
t=4 \pi \zeta
$$

and $F_{g}^{\text {inst }}(t, m)$ is the worldsheet instanton part of the standard genus $g$ topological string free energy.

To obtain the 't Hooft expansion of the fermionic trace and make contact with (2.60), we have to calculate the integral in (3.14), by doing a saddle-point expansion for $\hbar$ large. Let us denote by

$$
\sum_{g \geq 0} \mathcal{F}_{g}^{\mathbb{F}_{0}}(\lambda, m) \hbar^{2-2 g}
$$

the asymptotic expansion of the logarithm of the integral in (3.14). At leading order, one finds

$$
\lambda=\frac{\partial J_{0}(\zeta, m)}{\partial \zeta},
$$

which determines the 't Hooft parameter $\lambda$ as a function of $\zeta$, and conversely, $\zeta$ as a function of $\lambda$. The genus zero free energy $\mathcal{F}_{0}^{\mathbb{F}_{0}}(\lambda, m)$ is then given by a Legendre transform,

$$
\mathcal{F}_{0}^{\mathbb{F}_{0}}(\lambda, m)=J_{0}(\zeta(\lambda), m)-\lambda \zeta(\lambda)
$$

In particular

$$
\frac{\partial \mathcal{F}_{0}^{\mathbb{F}_{0}}}{\partial \lambda}=-\zeta
$$

The next-to-leading order correction to the saddle-point, $\mathcal{F}_{1}^{\mathbb{F}_{0}}(\lambda, m)$, is given by,

$$
\mathcal{F}_{1}^{F_{0}}(\lambda, m)=J_{1}(\zeta(\lambda), m)-\frac{1}{2} \log \left(2 \pi \frac{\partial^{2} J_{0}}{\partial \zeta^{2}}\right) .
$$

The higher order corrections can be computed systematically using the results of [57] (already exploited in this context in [13]): in the saddle-point approximation, the integral in (3.14) implements a transformation from the large radius frame, appropriate to $J_{g}(\zeta, m)$, to the conifold frame. Therefore, the functions $\mathcal{F}_{g}^{\mathbb{F}_{0}}(\lambda, m)$ appearing in (3.29) are the topological string free energies of local $\mathbb{P}^{1} \times \mathbb{P}^{1}$ in the conifold frame. According to the conjecture of $[1]$, they should be equal to the matrix model free energies appearing in (2.60)

$$
\mathcal{F}_{g}(\lambda, m)=\mathcal{F}_{g}^{\mathbb{F}_{0}}(\lambda, m), \quad g \geq 0
$$

This was tested in detail for local $\mathbb{P}^{2}$ and for local $\mathbb{F}_{2}$ (for a fixed valued of $\left.m_{\mathbb{F}_{2}}\right)$ in [13]. We will devote the rest of this paper to an explicit verification of (3.34), and in the next section we will compute the r.h.s. of (3.34) using standard techniques in topological string theory. 


\subsection{Topological Strings on Local $\mathbb{P}^{1} \times \mathbb{P}^{1}$}

Let us review some basic facts about the special geometry of local $\mathbb{P}^{1} \times \mathbb{P}^{1}$. Since this has two two-cycles, we can regard it as a two parameter model, and its mirror has then two complex moduli $z_{1}, z_{2}$. However, it has been known for some time that $m=z_{1} / z_{2}$ does not receive quantum corrections, therefore it should be rather regarded as a parameter (see for example [30]). We will then have a complex modulus, $z=z_{2}$, and a "mass" parameter $m$. The periods will be obtained as solutions to a single Picard-Fuchs (PF) equation corresponding to the operator [30]:

$$
\begin{aligned}
\mathcal{L}= & \left(8(1-m)^{2} z^{2}-4(1+m) z+\frac{1}{2}\right) \theta^{3}+\left(16(1-m)^{2} z^{2}-4(1+m) z\right) \theta^{2} \\
& +\left(6(1-m)^{2} z^{2}-(1+m) z\right) \theta
\end{aligned}
$$

where

$$
\theta=z \frac{\mathrm{d}}{\mathrm{d} z}
$$

This is the form of the operator which is appropriate for the large radius point $z=0$. As usual in local mirror symmetry, there will be a constant solution 1 , a logarithmic solution

$$
g_{1}(z)=\log (z)+\sigma_{1}(z)
$$

and a double logarithmic solution,

$$
g_{2}(z)=\log ^{2}(z)+2 \log (z) \sigma_{1}(z)+\sigma_{2}(z) .
$$

In these equations, $\sigma_{1,2}(z)$ are power series around $z=0$, whose coefficients depend on $m$. The very first orders read,

$$
\begin{aligned}
\sigma_{1}(z)= & 2\left(m^{\frac{1}{2}}+m^{-\frac{1}{2}}\right) m^{\frac{1}{2}} z+3\left(\left(m+m^{-1}\right)+4\right) m z^{2} \\
& +\frac{20}{3}\left(\left(m^{\frac{3}{2}}+m^{-\frac{3}{2}}\right)+9\left(m^{\frac{1}{2}}+m^{-\frac{1}{2}}\right)\right) m^{\frac{3}{2}} z^{3}+\mathcal{O}\left(z^{4}\right), \\
\sigma_{2}(z)= & 4\left(m^{\frac{1}{2}}+m^{-\frac{1}{2}}\right) m^{\frac{1}{2}} z+\left(13\left(m+m^{-1}\right)+40\right) m z^{2} \\
& +\frac{8}{9}\left(41\left(m^{\frac{3}{2}}+m^{-\frac{3}{2}}\right)+279\left(m^{\frac{1}{2}}+m^{-\frac{1}{2}}\right)\right) m^{\frac{3}{2}} z^{3}+\mathcal{O}\left(z^{4}\right)
\end{aligned}
$$

Let us now consider the following linear combinations of the basic periods,

$$
\begin{aligned}
& \Pi_{A}^{(\mathrm{lr})}(z)=\left(\begin{array}{lll}
0 & 1 & 0
\end{array}\right)\left(\begin{array}{l}
1 \\
g_{1}(z) \\
g_{2}(z)
\end{array}\right) \\
& \Pi_{B}^{(\mathrm{lr})}(z)=\left(\begin{array}{lll}
0 & \frac{\log m}{2} & \frac{1}{2}
\end{array}\right)\left(\begin{array}{l}
1 \\
g_{1}(z) \\
g_{2}(z)
\end{array}\right) .
\end{aligned}
$$

The first A-period determines the flat coordinate $t$ through the mirror map, while the second, B-period determines the genus zero free energy $F_{0}(t, m)$ at large radius, 


$$
-t=\Pi_{A}^{(\mathrm{lr})}, \quad \frac{\partial F_{0}}{\partial t}=\Pi_{B}^{(\mathrm{lr})} .
$$

After integration, we get,

$$
\begin{aligned}
& F_{0}(t, m) \\
& =\frac{t^{3}}{6}-\frac{\log m}{4} t^{2}-2\left(m^{\frac{1}{2}}+m^{-\frac{1}{2}}\right) m^{\frac{1}{2}} \mathrm{e}^{-t}-\frac{1}{4}\left(\left(m+m^{-1}\right)+16\right) m \mathrm{e}^{-2 t} \\
& \quad-\frac{2}{27}\left(\left(m^{\frac{3}{2}}+m^{-\frac{3}{2}}\right)+81\left(m^{\frac{1}{2}}+m^{-\frac{1}{2}}\right)\right) m^{\frac{3}{2}} \mathrm{e}^{-3 t}+\mathcal{O}\left(\mathrm{e}^{-4 t}\right) .
\end{aligned}
$$

Equivalently, one can obtain the same information from the equations

$$
\begin{gathered}
\frac{\partial t}{\partial z}=-\frac{2}{\pi z \sqrt{1-4(\sqrt{m}+1)^{2} z}} \mathrm{~K}\left(\frac{16 \sqrt{m} z}{4(\sqrt{m}+1)^{2} z-1}\right), \\
\frac{\partial^{2} F_{0}}{\partial t \partial z}=-\frac{2}{z \sqrt{1-4(\sqrt{m}-1)^{2} z}} \mathrm{~K}\left(\frac{4(\sqrt{m}+1)^{2} z-1}{4(\sqrt{m}-1)^{2} z-1}\right),
\end{gathered}
$$

which can be obtained from the results of [58] for local $\mathbb{F}_{2}$, together with the dictionary relating the moduli of local $\mathbb{F}_{2}$ to those of local $\mathbb{F}_{0}$.

We now analyze the theory near the conifold locus given by the vanishing of the discriminant:

$$
\begin{aligned}
\Delta & =1-8(m+1) z+16(m-1)^{2} z^{2} \\
& =\left(4(1+\sqrt{m})^{2} z-1\right)\left(4(1-\sqrt{m})^{2} z-1\right) .
\end{aligned}
$$

Note that there are two different branches of the conifold locus, related to the two square roots of $m$. For each value of $m$, we have a different conifold point in each of the branches of the conifold locus, and we have to analyze the topological string near an arbitrary point, as a function of $m$. For $m=1$, the topological string near the corresponding conifold point at $z=1 / 16$ has been analyzed in $[13,24,59]$. We will pick for convenience the branch of positive roots, and introduce the local variable,

$$
y=1-4(1+\sqrt{m})^{2} z,
$$

which vanishes at the conifold point

$$
z_{c}=\frac{1}{4(1+\sqrt{m})^{2}} .
$$

In the variables appropriate to the conifold point, the PF operator becomes

$$
\begin{aligned}
\tilde{\mathcal{L}}= & 4(y-1)^{2}\left(y(\mu-1)^{2}+4 \mu\right) \theta_{y}^{3} \\
& +4(y-1)\left(2 y^{2}(\mu-1)^{2}+y(1+\mu)^{2}+8 \mu\right) \theta_{y}^{2} \\
& +\left(3 y^{3}(\mu-1)^{2}+4 y^{2} \mu+y\left(\mu^{2}-6 \mu+1\right)+16 \mu\right) \theta_{y} .
\end{aligned}
$$

There is a basis of solutions given by a constant solution 1, a vanishing solution

$$
f_{1}(y)=y+\mathcal{O}\left(y^{2}\right)
$$


and a logarithmic solution

$$
f_{2}(y)=\log (y) f_{1}(y)+s(y), \quad s(y)=y+\mathcal{O}\left(y^{2}\right) .
$$

It is easy to solve for $f_{1}(y)$ and $s(y)$ as power series in $y$ with $m$-dependent coefficients:

$$
\begin{aligned}
f_{1}(y) & =y-\frac{\cosh (2 \xi)-11}{16} y^{2}+\frac{9 \cosh (4 \xi)-124 \cosh (2 \xi)+827}{1536} y^{3}+\mathcal{O}\left(y^{4}\right), \\
s(y) & =y-\frac{7 \cosh (2 \xi)-45}{32} y^{2}+\frac{27 \cosh (4 \xi)-380 \cosh (2 \xi)+1561}{1152} y^{3}+\mathcal{O}\left(y^{4}\right),
\end{aligned}
$$

where we expressed the results in terms of the variable $\xi$, related to $m$ by (3.17). The analytic continuation of the large radius periods to the conifold point must be a linear combination of the two solutions $f_{1}(y), f_{2}(y)$ found above. By expanding the exact results (3.44)-(3.45) around the conifold locus, one finds

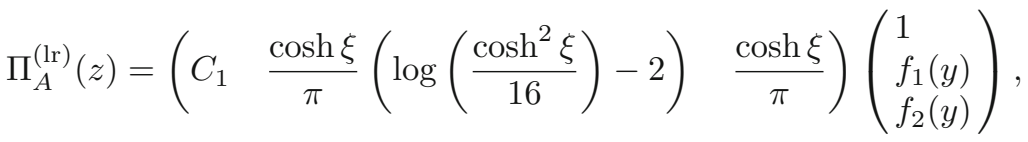

$$
\begin{aligned}
& \Pi_{B}^{(\mathrm{lr})}(z)=\left(\begin{array}{lll}
C_{2} & \pi \cosh \xi & 0
\end{array}\right)\left(\begin{array}{l}
1 \\
f_{1}(y) \\
f_{2}(y)
\end{array}\right)
\end{aligned}
$$

where $C_{1}, C_{2}$ are a priori $\xi$-dependent constants which we do not know how to evaluate analytically. These constants are given by the values of the large radius periods at the conifold point, i.e.,

$$
C_{1}=\Pi_{A}^{(\mathrm{lr})}\left(z_{c}\right), \quad C_{2}=\Pi_{B}^{(\mathrm{lr})}\left(z_{c}\right) .
$$

The constant $C_{1}$ can be computed analytically, and we present this computation in Appendix B. The constant $C_{2}$ can be calculated numerically, by evaluating the series (3.39) at the conifold point (where the series still converges). However, as we will see in the next section, the value of these constants is predicted by the conjecture of [1], and we will find a precise agreement with the analytical and numerical evaluations of $C_{1,2}$, respectively.

The above results determine the genus zero free energy at large radius $F_{0}(t, m)$. The genus one free energy can be obtained, for example, from the result for local $\mathbb{F}_{2}$ in [58], using the map of moduli. One finds,

$$
\begin{aligned}
F_{1}(t, m)= & -\frac{1}{12} \log \left(m^{\frac{1}{2}} z^{7}\left(16(m-1)^{2} z^{2}-8(m+1) z+1\right)\right) \\
& -\frac{1}{2} \log \left(-\frac{\partial t}{\partial z}\right),
\end{aligned}
$$

with the large radius expansion

$$
F_{1}(t, m)=-\frac{1}{24} \log (m)+\frac{t}{12}-\frac{1}{6}(1+m) \mathrm{e}^{-t}+\cdots
$$

The higher genus free energies near the conifold point can be obtained by integrating the holomorphic anomaly equation. A systematic computation 
has only been performed at $m=1[24,59]$, but it will provide us with useful tests, as we will see in the next section.

\subsection{Comparison}

Let us now compare the results from the matrix model/spectral theory side, with the predictions from the conjecture of [1]. We start with the genus zero free energy. By the expansion (3.43), we can write

$$
J_{0}\left(\zeta=\frac{t}{4 \pi}, \xi\right)=\frac{1}{16 \pi^{4}}\left(F_{0}(t, m)-\frac{\pi^{2}}{3} t+16 \pi^{4} A_{0}(\xi)\right),
$$

where $t$ is related to $\zeta$ by (3.28) and it is the standard Kähler parameter of the geometry. We also recall that $m$ is related to $\xi$ by (3.17). The 't Hooft parameter is given by $(3.30)$, which reads in this case,

$$
4 \pi^{3} \lambda=\Pi_{B}^{(\mathrm{lr})}-\frac{\pi^{2}}{3} .
$$

The form of the matrix model expansion (2.62) indicates that $\lambda$ must be a vanishing period at the conifold point, i.e.,

$$
\Pi_{B}^{(\mathrm{lr})}\left(z=z_{c}\right)=C_{2}=\frac{\pi^{2}}{3} .
$$

This gives a prediction for the value of the constant $C_{2}$, which we have verified by evaluating this constant numerically. This test involves doing a high precision numerical sum of the large radius expansion of $\Pi_{B}^{(\mathrm{lr})}\left(z=z_{c}\right)$, for different values of $m$.

We should note that, as far as we know, the constants that have to be added to the $B$-periods to obtain a vanishing period at the conifold point are not known a priori, and they have to be determined on a case-by-case basis, often numerically (see $[60,61]$ for some examples.) According to the conjecture of [1], this constant is obtained from the terms in the modified grand potential which are linear in the chemical potential and next-to-leading in $\hbar$. On the other hand, it follows from the general construction in [1] that these terms are in turn determined by the linear terms in the moduli appearing in the large radius free energy $F_{1}^{\mathrm{NS}}$. Therefore, we have the following consequence of the conjecture of [1]: in a toric CY threefold, the constant terms which appear in the vanishing periods at the conifold are determined by the coefficients of the linear terms in $F_{1}^{\mathrm{NS}}$. This is, of course, also the case for the example of local $\mathbb{P}^{2}$ studied in [13], and provides an intriguing link between refined genus one free energies and the special geometry of the conifold point.

Let us now proceed to compute $\mathcal{F}_{0}^{\mathbb{F}_{0}}$ from (3.32), which reads

$$
\frac{\partial \mathcal{F}_{0}^{\mathbb{F}_{0}}}{\partial \lambda}=\frac{1}{4 \pi} \Pi_{A}^{(\mathrm{lr})}
$$


This can be integrated to find, up to an integration constant,

$$
\begin{aligned}
\mathcal{F}_{0}^{\mathbb{F}_{0}}(\lambda, \xi)= & \frac{\lambda^{2}}{2}\left(\log \left(\frac{\pi^{2} \lambda \cosh \xi}{4}\right)-\frac{3}{2}\right)+\frac{C_{1}(\xi)}{4 \pi} \lambda+\pi^{2} \frac{1-3 \cosh (2 \xi)}{24 \cosh (\xi)} \lambda^{3} \\
& +\pi^{4} \frac{-73+68 \cosh (2 \xi)+45 \cosh (4 \xi)}{2304 \cosh ^{2}(\xi)} \lambda^{4} \\
& +\pi^{6} \frac{534-203 \cosh (2 \xi)-390 \cosh (4 \xi)-165 \cosh (6 \xi)}{30720 \cosh ^{3}(\xi)} \lambda^{5}+\mathcal{O}\left(\lambda^{6}\right) .
\end{aligned}
$$

The $\lambda$-independent function $C_{1}(\xi)$ appearing here is the one appearing in (3.54). This result agrees with the results in (2.62), (2.64), and (2.86) obtained in the matrix model, provided that

$$
C_{1}(\xi)=-\frac{8}{\pi} \operatorname{Im}\left(\operatorname{Li}_{2}\left(\mathrm{ie}^{\xi}\right)\right)
$$

Note that, as in the examples of [13], the r.h.s. of (3.63) is a prediction of the conjecture of [1] on the value of the $A$-period at the conifold point. This prediction comes from the explicit form of the potential (2.59), which is, in turn, determined by the explicit form of the integral kernel (2.22). As shown in Appendix B, the value (3.63) agrees precisely with the analytic evaluation of the A-period at the conifold.

Finally, we note that, according to the result (2.62) in the matrix model, the integration constant in $\mathcal{F}_{0}^{\mathbb{F}_{0}}(\lambda, m)$ should vanish. This implies that

$$
0=\mathcal{F}_{0}^{\mathbb{F}_{0}}(\lambda=0, m)=\left.\left(J_{0}(\zeta(\lambda), m)-\lambda \zeta(\lambda, m)\right)\right|_{\lambda=0} .
$$

Using (3.58), this yields the following relation between the function $A_{0}(\xi)$ in (3.23) and the value of the genus zero free energy at the conifold point:

$$
A_{0}(\xi)=\frac{1}{16 \pi^{4}}\left(F_{0}\left(-C_{1}(\xi), \xi\right)+\frac{\pi^{2}}{3} C_{1}(\xi)\right)
$$

where we used that $t\left(z_{c}\right)=-C_{1}(\xi)$. This is yet another remarkable consequence of the conjecture of [1] for the special geometry of the conifold point, and we have verified it numerically with high precision. To give a flavor of the validity of (3.65), in Fig. 3 we show the value of $A_{0}(\xi)$, as given in (3.23), against a numerical evaluation of the r.h.s. of (3.65) for some values of $\xi$.

We conclude that, at the level of the genus zero free energy, there is a remarkable agreement between the result obtained from the matrix model (i.e., from the spectral theory side) and the predictions of [1] based on topological string theory. In particular, one can use the results of the matrix model/spectral theory side to obtain non-trivial information about the conifold theory which is not available otherwise (as far as we know): the analytic values of the periods and the genus zero free energy at the conifold point.

Let us now consider the genus one free energy. Note that

$$
J_{1}(\zeta, \xi)=\frac{\pi}{3} \zeta+A_{1}(\xi)+F_{1}^{\text {inst }}(t, \xi)=A_{1}(\xi)+\frac{\xi}{6}+F_{1}(t, \xi),
$$




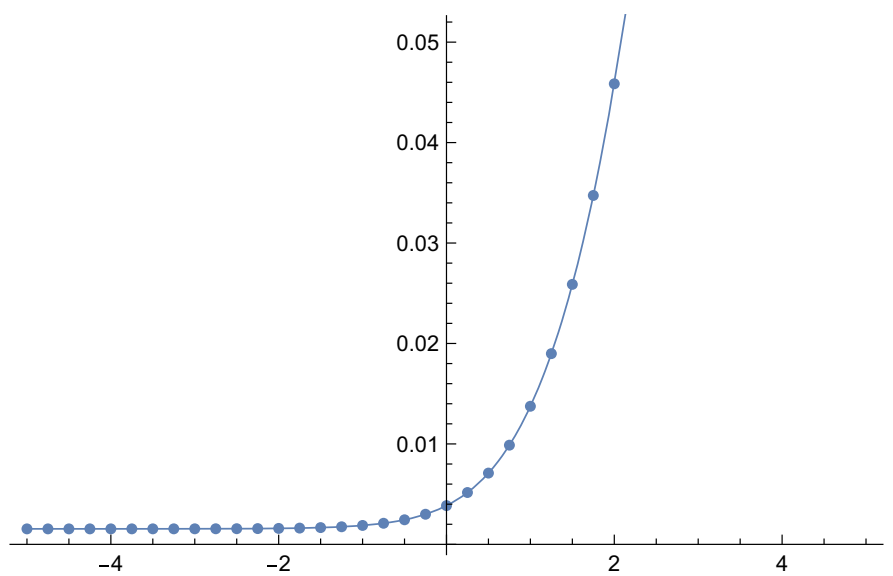

Figure 3. The continuous line shows the exact function $A_{0}(\xi)$, as given in (3.23), while the dots are numerical evaluations of the r.h.s. of (3.65) for some values of $\xi$

where we used (3.56) and the expansion (3.57). If we now take into account (3.33) and the equation

$$
\frac{\partial^{2} J_{0}(\zeta)}{\partial \zeta^{2}}=4 \pi \frac{\partial \lambda}{\partial t},
$$

we obtain

$$
\begin{aligned}
\mathcal{F}_{1}^{\mathbb{F}_{0}}(\lambda)= & A_{1}(\xi)+\frac{\xi}{6}-\frac{1}{24} \log m \\
& -\frac{1}{12} \log \left(m^{\frac{1}{2}} z^{7}(\lambda)\left(16(m-1)^{2} z^{2}(\lambda)-8(m+1) z(\lambda)+1\right)\right) \\
& +\frac{1}{2} \log \left(-\frac{1}{8 \pi^{2}} \frac{\partial z(\lambda)}{\partial \lambda}\right) .
\end{aligned}
$$

By the explicit expression for $A_{1}(\xi)$ in (3.23), we find that the small $\lambda$ expansion of this function is,

$$
\begin{aligned}
\mathcal{F}_{1}^{\mathbb{F}_{0}}(\lambda)= & -\frac{1}{12} \log \lambda-\frac{1}{12} \log \hbar+\zeta^{\prime}(-1) \\
& +\pi^{2} \frac{-1+3 \cosh (2 \xi)}{48 \cosh ^{2}(\xi)} \lambda \\
& +\pi^{4} \frac{127+4 \cosh (2 \xi)-27 \cosh (4 \xi)}{2304 \cosh ^{2}(\xi)} \lambda^{2}+\mathcal{O}\left(\lambda^{3}\right),
\end{aligned}
$$

which is in precise agreement with what was found in (2.62), (2.65).

There are some non-trivial tests that can be done at higher genus, in the case $\xi=0$, using the results in $[24,59]$. Let us first recall that the topological string free energies in the conifold frame, when expanded around the conifold point in terms of a vanishing period, have a universal critical behavior characterized by a pole of order $2 g-2$, for $g \geq 2[62]$. It was then pointed out 
in [63] that the full expansion satisfies a "gap condition", i.e., after this pole, the rest of the expansion is regular and it starts at zeroth order in the vanishing period. This has been exploited to constrain solutions to the holomorphic anomaly equations. However, in the matrix model free energies, as one can see in (2.62), the expansion around the conifold fulfills a "strong" gap condition, in the sense that the expansion in $\lambda$ after the pole starts at first order in $\lambda$ (and not at zeroth order). In contrast, the conventional topological string free energies satisfy only a "weak" gap condition. In practice, this has the following consequence. Let us consider the instanton part of the large radius, genus $g$ free energies $F_{g}^{\text {inst }}(t, m)$, and let us perform a symplectic transformation to the conifold frame. The "weak" gap condition of [63] implies that the expansion of the resulting quantities around the conifold point is of the form,

$$
\frac{B_{2 g}}{2 g(2 g-2)} t_{c}^{2-2 g}+b_{g}(\xi)+\mathcal{O}\left(t_{c}\right)
$$

where $t_{c}=4 \pi^{2} \lambda$ is a vanishing period at the conifold. ${ }^{3}$ Then, it follows from the last line in (3.27) that

$$
\mathcal{F}_{g}(\lambda, \xi)=\frac{B_{2 g}}{2 g(2 g-2)} \lambda^{2-2 g}+\left(4 \pi^{2}\right)^{2 g-2} b_{g}(\xi)+A_{g}(\xi)+\mathcal{O}(\lambda) .
$$

Therefore, consistency with the expansion (2.62), which satisfies a strong gap condition, requires that

$$
b_{g}(\xi)=-\frac{A_{g}(\xi)}{\left(4 \pi^{2}\right)^{2 g-2}}, \quad g \geq 2 .
$$

This can be regarded as yet another prediction of spectral theory for the topological string, since the coefficients $A_{g}(\xi)$ have been fixed by consistency with studies of the spectrum. For $\xi=0$, the constants $b_{g}(0)$ can be computed systematically from the holomorphic anomaly equations [24,59]. One finds, for the very first genera,

$$
b_{2}(0)=-\frac{1}{1152}, \quad b_{3}(0)=\frac{23}{5806080}, \quad b_{4}(0)=-\frac{19}{278691840},
$$

and using (3.24), one verifies that (3.72) is indeed satisfied.

Finally, we note that the genus two free energy in the conifold frame is given by $[24,59]$,

$$
F_{2}^{\text {inst }}\left(t_{c}, \xi=0\right)=-\frac{1}{240 t_{c}^{2}}-\frac{1}{1152}+\frac{53 t_{c}}{122880}-\frac{2221 t_{c}^{2}}{14745600}+\cdots
$$

The third term in this expansion agrees with the coefficient $f_{2,1}$ in $(2.65)$, for $\xi=0$ after taking into account the overall factor $\left(4 \pi^{2}\right)^{2}$ in $(3.27)$. We conclude that the ' $t$ Hooft expansion of the fermionic traces, as calculated by the matrix model, is in perfect agreement with the predictions of [1] (and with the result of (Hatsuda, Y.: Unpublished) for the function $A\left(m_{\mathbb{F}_{0}}, \hbar\right)$ ).

\footnotetext{
${ }^{3}$ We are considering just the instanton part of the large radius free energies, so we are not including the constant map contribution to these amplitudes to zero. Note, however, that adding this contribution does not lead in general to a strong gap condition at the conifold. In other words, $-b_{g}$ is not the constant map contribution at large radius.
} 


\section{Conclusions and Open Problems}

In this paper we have found an explicit expression for the integral kernel of the trace class operator associated to the mirror curve of local $\mathbb{F}_{0}$, for arbitrary values of the mass parameter. This makes it possible to obtain a matrix model computing the fermionic spectral traces of this operator. This model turns out to be an $O(2)$ model, which can be exactly solved in the planar limit. Using this matrix model, we have verified in detail that the fermionic spectral traces of (2.20) provide a non-perturbative definition of the topological string on this geometry, in the sense that their asymptotic 't Hooft expansion is given by the genus expansion of the topological string. This provides yet another test of the conjecture in [1]. In particular, our calculation checks the conjectural form of the quantum-mechanical instanton corrections to the spectral problem proposed in [1].

There are various obvious problems raised by our results. It would be interesting to improve our checks by calculating higher genus amplitudes directly in the matrix model, although this type of calculations is not simple for $O(n)$ models. Even at genus zero, it would be interesting to have an analytic proof that the function $\mathcal{F}_{0}$ obtained in the matrix model agrees exactly with the genus zero free energy of the topological string $\mathcal{F}_{0}^{\mathbb{F}_{0}}$. Another obvious open problem is to extend this type of calculations to other geometries, like for example local $\mathcal{B}_{n}$, where $\mathcal{B}_{n}$ is the blow-up of $\mathbb{P}^{2}$ at $n$ points. To do this, we would need an explicit form for the integral kernels of the corresponding trace class operators. It would be also interesting to find exponentially small corrections to the 't Hooft expansion of the matrix model studied here, to construct a trans-series expansion of the matrix model free energy, in the spirit of [64] (see [65] for a recent, detailed case study of trans-series in the quartic matrix model). This could then be compared to the predictions of [1] and/or to the trans-series construction of $[66,67]$.

Another research direction concerns the field theory limit of the model analyzed in this paper. It can be explicitly shown that the spectral problem for the operator (2.10) has a double-scaling limit in which one recovers the quantum spectral curve of $S U(2)$ Toda given in [68]. This corresponds to the field theory limit of the topological string, which is pure $N=2$ Yang-Mills theory [32]. According to [3], the NS limit of the instanton partition function of [69] provides a quantization condition for this spectral problem. This can be verified using the perturbative WKB approach [5], but there are also nonperturbative corrections (see [70-72] for different perspectives on this issue). It would be interesting to analyze this field theory limit using the tools introduced here.

Finally, as we have explained, the matrix model in this paper generalizes the ABJ(M) matrix model, and in particular extends it to arbitrary values of $M$. This is due to the fact that the dependence on $M$ is through the mass parameter $m_{\mathbb{F}_{0}}$, as shown in (2.102). In contrast, in the existing matrix models for ABJ theory $[21,46], M$ has to be in principle a positive integer. This might be useful to relate ABJ theory to higher spin theories $[73,74]$. 


\section{Appendix A. The Quantum Dilogarithm}

The quantum dilogarithm $\Phi_{\mathrm{b}}(x)$ is defined by [33-35]

$$
\Phi_{\mathrm{b}}(x)=\frac{\left(\mathrm{e}^{2 \pi \mathrm{b}\left(x+c_{\mathrm{b}}\right)} ; q\right)_{\infty}}{\left(\mathrm{e}^{2 \pi \mathrm{b}^{-1}\left(x-c_{\mathrm{b}}\right)} ; \tilde{q}\right)_{\infty}},
$$

where

$$
q=\mathrm{e}^{2 \pi \mathrm{ib}^{2}}, \quad \tilde{q}=\mathrm{e}^{-2 \pi \mathrm{ib}^{-2}}, \quad \operatorname{Im}\left(\mathrm{b}^{2}\right)>0
$$

and

$$
c_{\mathrm{b}}=\frac{\mathrm{i}}{2}\left(\mathrm{~b}+\mathrm{b}^{-1}\right) .
$$

An integral representation in the strip $|\operatorname{Im} z|<\left|\operatorname{Im} c_{\mathrm{b}}\right|$ is given by

$$
\Phi_{\mathrm{b}}(x)=\exp \left(\int_{\mathbb{R}+\mathrm{i} \epsilon} \frac{\mathrm{e}^{-2 \mathrm{i} x z}}{4 \sinh (z \mathrm{~b}) \sinh \left(z \mathrm{~b}^{-1}\right)} \frac{\mathrm{d} z}{z}\right) .
$$

Remarkably, this function admits an extension to all values of $\mathrm{b}$ with $\mathrm{b}^{2} \notin \mathbb{R}_{\leq 0}$. $\Phi_{\mathrm{b}}(x)$ is a meromorphic function of $x$ with

$$
\text { poles: } c_{\mathrm{b}}+\mathrm{i} \mathbb{N b}+\mathrm{iNb}^{-1}, \quad \text { zeros: }-c_{\mathrm{b}}-\mathrm{i} \mathbb{N b}-\mathrm{i} \mathbb{N b}^{-1} \text {. }
$$

The functional equation

$$
\Phi_{\mathrm{b}}(x) \Phi_{\mathrm{b}}(-x)=\mathrm{e}^{\pi \mathrm{i} x^{2}} \Phi_{\mathrm{b}}(0)^{2}, \quad \Phi_{\mathrm{b}}(0)=\left(\frac{q}{\tilde{q}}\right)^{\frac{1}{48}}=\mathrm{e}^{\pi \mathrm{i}\left(\mathrm{b}^{2}+\mathrm{b}^{-2}\right) / 24},
$$

allows us to move $\Phi_{\mathrm{b}}(x)$ from the denominator to the numerator. In addition, when $b$ is either real or on the unit circle, we have the unitarity relation

$$
\overline{\Phi_{\mathrm{b}}(x)}=\frac{1}{\Phi_{\mathrm{b}}(\bar{x})} .
$$

The asymptotics of the quantum dilogarithm are given by [75]

$$
\Phi_{\mathrm{b}}(x) \sim \begin{cases}\Phi_{\mathrm{b}}(0)^{2} \mathrm{e}^{\pi \mathrm{i} x^{2}} & \text { when } \Re(x) \gg 0, \\ 1 & \text { when } \Re(x) \ll 0 .\end{cases}
$$

The quantum dilogarithm is a quasi-periodic function. Explicitly, it satisfies the equations

$$
\begin{aligned}
\frac{\Phi_{\mathrm{b}}\left(x+c_{\mathrm{b}}+\mathrm{ib}\right)}{\Phi_{\mathrm{b}}\left(x+c_{\mathrm{b}}\right)} & =\frac{1}{1-q \mathrm{e}^{2 \pi \mathrm{b} x}} \\
\frac{\Phi_{\mathrm{b}}\left(x+c_{\mathrm{b}}+\mathrm{ib}^{-1}\right)}{\Phi_{\mathrm{b}}\left(x+c_{\mathrm{b}}\right)} & =\frac{1}{1-\tilde{q}^{-1} \mathrm{e}^{2 \pi \mathrm{b}^{-1} x}} .
\end{aligned}
$$

When $b$ is small, we can use the following asymptotic expansion (see for example $[75,76])$,

$$
\log \Phi_{\mathrm{b}}\left(\frac{x}{2 \pi \mathrm{b}}\right) \sim \sum_{k=0}^{\infty}\left(2 \pi \mathrm{ib}^{2}\right)^{2 k-1} \frac{B_{2 k}(1 / 2)}{(2 k) !} \mathrm{Li}_{2-2 k}\left(-\mathrm{e}^{x}\right),
$$

where $B_{2 k}(z)$ is the Bernoulli polynomial. 


\section{Appendix B. The A-Period at the Conifold Point}

In this short Appendix we compute the A-period $\Pi_{A}^{(\mathrm{lr})}(z)$ at the conifold point $z=z_{c}$, for arbitrary values of $m$ (or, equivalently, of $\xi$ ). The starting point of this calculation is the integral

$$
\mathcal{I}=\frac{1}{(2 \pi \mathrm{i})^{2}} \int_{\mathbb{S}^{1} \times \mathbb{S}^{1}} \log P(z, w) \frac{\mathrm{d} z}{z} \frac{\mathrm{d} w}{w},
$$

where

$$
P(z, w)=2\left(x^{2}+y^{2}\right)+x^{2}\left(z+z^{-1}\right)+2 y^{2}\left(w+w^{-1}\right) .
$$

Note that $P(z, w)$ is essentially the polynomial defining the mirror curve to local $\mathbb{F}_{0}$, and the integral $\mathcal{I}$ is the logarithmic Mahler measure of this polynomial. Let us define

$$
z_{1}=\frac{x^{4}}{4\left(x^{2}+y^{2}\right)^{2}}, \quad z_{2}=\frac{y^{4}}{4\left(x^{2}+y^{2}\right)^{2}} .
$$

By expanding $\log P(z, w)$ in power series in $z_{1,2}$, we find

$$
\mathcal{I}=\log \left(2\left(x^{2}+y^{2}\right)\right)-\sum_{n=1}^{\infty} \sum_{2 k+2 l=n} \frac{\Gamma(2 k+2 l)}{\Gamma(1+k)^{2} \Gamma(1+l)^{2}} z_{1}^{k} z_{2}^{l} .
$$

If we identify the variables $z_{1,2}$ with the moduli of local $\mathbb{F}_{0}$, we have that

$$
m=\frac{z_{1}}{z_{2}}=\left(\frac{x}{y}\right)^{4}
$$

and we finally obtain

$$
\mathcal{I}=2 \log y-\frac{1}{2} \Pi_{A}^{(\operatorname{lr})}\left(z_{c}\right)
$$

where $z_{c}$ is given in (3.48).

On the other hand, the integral (B.1) was explicitly computed by Kasteleyn in Sect. 3 of [77], in the analysis of the dimer model on the bipartite square lattice on the torus (see [78] for a nice summary of the subject.) By writing it as

$$
\mathcal{I}=\frac{1}{\pi^{2}} \int_{0}^{\pi} \int_{0}^{\pi} \log \left(4\left(x^{2} \cos ^{2} \omega+y^{2} \cos ^{2} \omega^{\prime}\right)\right) \mathrm{d} \omega \mathrm{d} \omega^{\prime},
$$

one can first perform the integral w.r.t. $\omega^{\prime}$, compute the remaining integral as a power series in $x / y=\mathrm{e}^{\xi}$, and resum the resulting series in terms of the dilogarithm. One finds,

$$
\mathcal{I}=2 \log y+\frac{4}{\pi} \operatorname{Im}\left(\operatorname{Li}_{2}\left(\mathrm{ie}^{\xi}\right)\right) .
$$

By comparing this to (B.6), we conclude that

$$
\Pi_{A}^{(\mathrm{lr})}\left(z_{c}\right)=-\frac{8}{\pi}\left(\mathrm{Li}_{2}\left(\mathrm{ie}^{\xi}\right)\right)
$$

which is precisely what we obtained from (3.63). 


\section{Acknowledgements}

We would like to thank David Cimasoni, Santiago Codesido, Ricardo CousoSantamaría, Alba Grassi, Jie Gu, Yasuyuki Hatsuda, Albrecht Klemm, Kazumi Okuyama, Jonas Reuter and Ricardo Schiappa for useful discussions and correspondence. We are particularly thankful to Ricardo Schiappa for a detailed reading of the draft. This work is supported in part by the Fonds National Suisse, subsidies 200020-141329, 200021-156995 and 200020141329, and by the NCCR 51NF40-141869 "The Mathematics of Physics" (SwissMAP).

\section{References}

[1] Grassi, A., Hatsuda, Y., Mariño, M.: Topological strings from quantum mechanics. arXiv:1410.3382 [hep-th].

[2] Aganagic, M., Dijkgraaf, R., Klemm, A., Mariño, M., Vafa, C.: Topological strings and integrable hierarchies. Commun. Math. Phys. 261, 451 (2006). arXiv:hep-th/0312085.

[3] Nekrasov, N.A., Shatashvili, S.L.: Quantization of integrable systems and four dimensional gauge theories. arXiv:0908.4052 [hep-th].

[4] Aganagic, M., Cheng, M.C.N., Dijkgraaf, R., Krefl, D., Vafa, C.: Quantum geometry of refined topological strings. JHEP 1211, 019 (2012). arXiv:1105.0630 [hepth].

[5] Mironov, A., Morozov, A.: Nekrasov functions and exact Bohr-Zommerfeld integrals. JHEP 1004, 040 (2010). arXiv:0910.5670 [hep-th].

[6] Mariño, M., Putrov, P.: ABJM theory as a Fermi gas. J. Stat. Mech. 1203, P03001 (2012). arXiv:1110.4066 [hep-th].

[7] Hatsuda, Y., Moriyama, S., Okuyama, K.: Instanton effects in ABJM theory from fermi gas approach. JHEP 1301, 158 (2013). arXiv:1211.1251 [hep-th].

[8] Hatsuda, Y., Moriyama, S., Okuyama, K.: Instanton bound states in ABJM theory. JHEP 1305, 054 (2013). arXiv:1301.5184 [hep-th].

[9] Hatsuda, Y., Mariño, M., Moriyama, S., Okuyama, K.: Non-perturbative effects and the refined topological string. JHEP 1409, 168 (2014). arXiv:1306.1734 [hepth].

[10] Kallen, J., Mariño, M.: Instanton effects and quantum spectral curves. arXiv:1308.6485 [hep-th].

[11] Codesido, S., Grassi, A., Mariño, M.: Exact results in $\mathcal{N}=8$ Chern-Simonsmatter theories and quantum geometry. JHEP 1507, 011 (2015). arXiv:1409.1799 [hep-th].

[12] Huang, M.X., Wang, X.F.: Topological strings and quantum spectral problems. JHEP 1409, 150 (2014). arXiv:1406.6178 [hep-th].

[13] Mariño, M., Zakany, S.: Matrix models from operators and topological strings. arXiv:1502.02958 [hep-th].

[14] Kashaev, R., Mariño, M.: Operators from mirror curves and the quantum dilogarithm. arXiv:1501.01014 [hep-th].

[15] Kostov, I.K.: Exact solution of the six vertex model on a random lattice. Nucl. Phys. B 575, 513 (2000). arXiv:hep-th/9911023. 
[16] Gu, J., Klemm, A., Mariño, M., Reuter, J.: Exact solutions to quantum spectral curves by topological string theory. JHEP 1510, 025 (2015). arXiv:1506.09176 [hep-th].

[17] Eynard, B., Kristjansen, C.: Exact solution of the $O(n)$ model on a random lattice. Nucl. Phys. B 455, 577 (1995). arXiv:hep-th/9506193.

[18] Eynard, B., Kristjansen, C.: More on the exact solution of the $O(n)$ model on a random lattice and an investigation of the case $|n|>2$. Nucl. Phys. B 466, 463 (1996). arXiv:hep-th/9512052.

[19] Grassi, A., Mariño, M.: M-theoretic matrix models. JHEP 1502, 115 (2015). arXiv:1403.4276 [hep-th].

[20] Iqbal, A., Kashani-Poor, A.K.: Instanton counting and Chern-Simons theory. Adv. Theor. Math. Phys. 7(3), 457 (2003). arXiv:hep-th/0212279.

[21] Awata, H., Hirano, S., Shigemori, M.: The partition function of ABJ theory. Prog. Theor. Exp. Phys. 053B04 (2013). arXiv:1212.2966.

[22] Honda, M.: Direct derivation of "mirror" ABJ partition function. JHEP 1312, 046 (2013). arXiv:1310.3126 [hep-th].

[23] Honda, M., Okuyama, K.: Exact results on ABJ theory and the refined topological string. JHEP 1408, 148 (2014). arXiv:1405.3653 [hep-th].

[24] Drukker, N., Mariño, M., Putrov, P.: From weak to strong coupling in ABJM theory. Commun. Math. Phys. 306, 511 (2011). arXiv:1007.3837 [hep-th].

[25] Aganagic, M., Klemm, A., Mariño, M., Vafa, C.: Matrix model as a mirror of Chern-Simons theory. JHEP 0402, 010 (2004). arXiv:hep-th/0211098.

[26] Gopakumar, R., Vafa, C.: On the gauge theory/geometry correspondence. Adv. Theor. Math. Phys. 3, 1415 (1999). arXiv:hep-th/9811131.

[27] Mariño, M.: Chern-Simons theory, matrix integrals, and perturbative three manifold invariants. Commun. Math. Phys. 253, 25 (2004). arXiv:hep-th/0207096.

[28] Halmagyi, N., Yasnov, V.: The Spectral curve of the lens space matrix model. JHEP 0911, 104 (2009). arXiv:hep-th/0311117.

[29] Halmagyi, N., Okuda, T., Yasnov, V.: Large N duality, lens spaces and the Chern-Simons matrix model. JHEP 0404, 014 (2004). arXiv:hep-th/0312145.

[30] Huang, M.X., Klemm, A., Poretschkin, M.: Refined stable pair invariants for E-, M- and $[p, q]$-strings. JHEP 1311, 112 (2013). arXiv:1308.0619 [hep-th].

[31] Huang, M.X., Klemm, A., Reuter, J., Schiereck, M.: Quantum geometry of del Pezzo surfaces in the Nekrasov-Shatashvili limit. JHEP 1502, 031 (2015). arXiv:1401.4723 [hep-th].

[32] Katz, S.H., Klemm, A., Vafa, C.: Geometric engineering of quantum field theories. Nucl. Phys. B 497, 173 (1997). arXiv:hep-th/9609239.

[33] Faddeev, L.D.: Discrete Heisenberg-Weyl group and modular group. Lett. Math. Phys. 34, 249 (1995)

[34] Faddeev, L.D., Kashaev, R.M.: Quantum dilogarithm. Modern Phys. Lett. A 9, 427 (1994)

[35] Faddeev, L.D., Kashaev, R.M., Volkov, A.Y.: Strongly coupled quantum discrete Liouville theory. 1. Algebraic approach and duality. Commun. Math. Phys. 219, 199 (2001). arXiv:hep-th/0006156. 
[36] Faddeev, L.D.: Current-like variables in massive and massless integrable models. In *Varenna 1994, Quantum groups and their applications in physics* 117135. arXiv:hep-th/9408041.

[37] Zamolodchikov, A.B.: Painlevé III and 2-d polymers. Nucl. Phys. B 432, 427 (1994). arXiv:hep-th/9409108.

[38] Tracy, C.A., Widom, H.: Proofs of two conjectures related to the thermodynamic Bethe ansatz. Commun. Math. Phys. 179, 667 (1996). arXiv:solv-int/9509003.

[39] Simon, B.: Trace Ideals and Their Applications, 2nd edn. American Mathematical Society, Providence (2000)

[40] Kapustin, A., Willett, B., Yaakov, I.: Nonperturbative tests of three-dimensional dualities. JHEP 1010, 013 (2010). arXiv:1003.5694 [hep-th].

[41] Kostov, I.K.: $\mathrm{O}(n)$ vector model on a planar random lattice: spectrum of anomalous dimensions. Mod. Phys. Lett. A 4, 217 (1989)

[42] Kostov, I.K., Staudacher, M.: Multicritical phases of the $O(n)$ model on a random lattice. Nucl. Phys. B 384, 459 (1992). arXiv:hep-th/9203030.

[43] Abramowitz, M., Stegun, I.: Handbook of Mathematical Functions with Formulas, Graphs, and Mathematical Tables. Dover, New York (1964)

[44] Klebanov, I.R., Tseytlin, A.A.: Entropy of near extremal black p-branes. Nucl. Phys. B 475, 164 (1996). arXiv:hep-th/9604089.

[45] Herzog, C.P., Klebanov, I.R., Pufu, S.S., Tesileanu, T.: Multi-matrix models and tri-sasaki Einstein spaces. Phys. Rev. D 83, 046001 (2011). arXiv:1011.5487 [hep-th].

[46] Kapustin, A., Willett, B., Yaakov, I.: Exact results for Wilson loops in superconformal Chern-Simons theories with matter. JHEP 1003, 089 (2010). arXiv:0909.4559 [hep-th].

[47] Aharony, O., Bergman, O., Jafferis, D.L., Maldacena, J.: N=6 superconformal Chern-Simons-matter theories, M2-branes and their gravity duals. JHEP 0810, 091 (2008). arXiv:0806.1218 [hep-th].

[48] Aharony, O., Bergman, O., Jafferis, D.L.: Fractional M2-branes. JHEP 0811, 043 (2008). arXiv:0807.4924 [hep-th].

[49] Matsumoto, S., Moriyama, S.: ABJ fractional brane from ABJM Wilson loop. JHEP 1403, 079 (2014). arXiv:1310.8051 [hep-th].

[50] Mariño, M., Putrov, P.: Exact results in ABJM theory from topological strings. JHEP 1006, 011 (2010). arXiv:0912.3074 [hep-th].

[51] Hanada, M., Honda, M., Honma, Y., Nishimura, J., Shiba, S., Yoshida, Y.: Numerical studies of the ABJM theory for arbitrary $\mathrm{N}$ at arbitrary coupling constant. JHEP 1205, 121 (2012). arXiv:1202.5300 [hep-th].

[52] Hatsuda, Y., Okuyama, K.: Probing non-perturbative effects in M-theory. JHEP 1410, 158 (2014). arXiv:1407.3786 [hep-th].

[53] Witten, E.: Quantum field theory and the Jones polynomial. Commun. Math. Phys. 121, 351 (1989)

[54] Hatsuda, Y., Okuyama, K.: Resummations and non-perturbative corrections. JHEP 1509, 051 (2015). arXiv:1505.07460 [hep-th].

[55] Mkrtchyan, R.L.: Nonperturbative universal Chern-Simons theory. JHEP 1309, 054 (2013). arXiv:1302.1507 [hep-th]. 
[56] Gopakumar, R., Vafa, C.: $\mathrm{M}$ theory and topological strings. 2. arXiv:hep-th/9812127.

[57] Aganagic, M., Bouchard, V., Klemm, A.: Topological strings and (almost) modular forms. Commun. Math. Phys. 277, 771 (2008). arXiv:hep-th/0607100.

[58] Brini, A., Tanzini, A.: Exact results for topological strings on resolved Y**p,q singularities. Commun. Math. Phys. 289, 205 (2009). arXiv:0804.2598 [hep-th].

[59] Haghighat, B., Klemm, A., Rauch, M.: Integrability of the holomorphic anomaly equations. JHEP 0810, 097 (2008). arXiv:0809.1674 [hep-th].

[60] Greene, B.R., Lazaroiu, C.I.: Collapsing D-branes in Calabi-Yau moduli space. 1. Nucl. Phys. B 604, 181 (2001). arXiv:hep-th/0001025.

[61] De la Ossa, X., Florea, B., Skarke, H.: D-branes on noncompact CalabiYau manifolds: K theory and monodromy. Nucl. Phys. B 644, 170 (2002). arXiv:hep-th/0104254.

[62] Ghoshal, D., Vafa, C.: $c=1$ string as the topological theory of the conifold. Nucl. Phys. B 453, 121 (1995). arXiv:hep-th/9506122.

[63] Huang, M.X., Klemm, A.: Holomorphic anomaly in Gauge theories and matrix models. JHEP 0709, 054 (2007). arXiv:hep-th/0605195.

[64] Mariño, M.: Nonperturbative effects and nonperturbative definitions in matrix models and topological strings. JHEP 0812, 114 (2008). arXiv:0805.3033 [hepth].

[65] Couso-Santamaria, R., Schiappa, R., Vaz, R.: Finite N from resurgent large N. Ann. Phys. 356, 1 (2015). arXiv:1501.01007 [hep-th].

[66] Couso-Santamaria, R., Edelstein, J.D., Schiappa, R., Vonk, M.: Resurgent transseries and the holomorphic anomaly. Ann. Henri Poincaré 17(2), 331 (2016). arXiv:1308.1695 [hep-th].

[67] Couso-Santamaria, R., Edelstein, J.D., Schiappa, R., Vonk, M.: Resurgent transseries and the holomorphic anomaly: nonperturbative closed strings in local $\mathbb{C P}^{2}$. Commun. Math. Phys. 338(1), 285 (2015). arXiv:1407.4821 [hep-th].

[68] Gaudin, M., Pasquier, V.: The periodic toda chain and a matrix generalization of the bessel function's recursion relations. J. Phys. A 25, 5243 (1992)

[69] Nekrasov, N.A.: Seiberg-Witten prepotential from instanton counting. Adv. Theor. Math. Phys. 7, 831 (2004). arXiv:hep-th/0206161.

[70] Krefl, D.: Non-Perturbative quantum geometry II. JHEP 1412, 118 (2014). arXiv:1410.7116 [hep-th].

[71] Basar, G., Dunne, G.V.: Resurgence and the Nekrasov-Shatashvili limit: connecting weak and strong coupling in the Mathieu and Lamé systems. JHEP 1502, 160 (2015). arXiv:1501.05671 [hep-th].

[72] Kashani-Poor, A.K., Troost, J.: Pure $\mathcal{N}=2$ super Yang-Mills and exact WKB. JHEP 1508, 160 (2015). arXiv:1504.08324 [hep-th].

[73] Chang, C.M., Minwalla, S., Sharma, T., Yin, X.: ABJ triality: from higher spin fields to strings. J. Phys. A 46, 214009 (2013). arXiv:1207.4485 [hep-th].

[74] Hirano, S., Honda, M., Okuyama, K., Shigemori, M.: ABJ theory in the higher spin limit. arXiv:1504.00365 [hep-th].

[75] Ellegaard Andersen, J., Kashaev, R.: A TQFT from quantum Teichmüller Theory. Commun. Math. Phys. 330, 887 (2014). arXiv:1109.6295 [math.QA]. 
[76] Mcintosh, R.J.: Some asymptotic formulae for q-shifted factorials. Ramanujan J. 3, 205 (1999)

[77] Kasteleyn, P.W.: The statistics of dimers on a lattice: I the number of dimer arrangements on a quadratic lattice. Physica 27, 1209 (1961)

[78] Cimasoni, D.: The geometry of dimer models. arXiv:1409.4631.

Rinat Kashaev and Marcos Mariño

Section de Mathématiques

Université de Genève

Genève, CH-1211, Switzerland

e-mail: rinat.kashaev@unige.ch;

marcos.marino@unige.ch

Marcos Mariño and Szabolcs Zakany

Département de Physique Théorique

Université de Genève

Genève, CH-1211, Switzerland

e-mail: szabolcs.zakany@unige.ch

Communicated by Boris Pioline.

Received: September 28, 2015.

Accepted: January 5, 2016. 